\title{
Diagonal Short Time Asymptotics of Heat Kernels for Certain Degenerate Second Order Differential Operators of Hiörmander Type
}

By

\author{
Satoshi TAKANOBU*
}

\section{Introduction}

Let $V_{0}, V_{1}, \cdots, V_{n} \in \mathbb{C}_{b}^{\infty}\left(\mathbb{R}^{d}, \mathbb{R}^{d}\right)$. Let $\hat{V}_{i} \in \mathfrak{X}\left(\mathbb{R}^{d}\right)$ be defined by $\hat{V}_{i}=$ $\sum_{j=1}^{d} V_{i}^{j} \frac{\partial}{\partial x^{j}}, i \in\{0,1, \cdots, n\}$, and let $L$ denote a second order differential operator written in Hörmander's form

$$
L=\frac{1}{2} \sum_{i=1}^{n} \hat{V}_{i}^{2}+\hat{V}_{0}
$$

Assume that at every $x \in \mathbb{R}^{d}, \hat{V}_{0}, \hat{V}_{1}, \cdots, \hat{V}_{n}$ satisfy the Hörmander condition: For some $\nu \geq 1$

$$
\left\{\begin{aligned}
\text { linear span }\left\{\left[\hat{V}_{i_{a}},\left[\hat{V}_{i_{a-1}},\left[\cdots,\left[\hat{V}_{i_{2}}, \hat{V}_{i_{1}}\right] \cdots\right](x) ; 1 \leq a \leq \nu\right.\right.\right. \\
\left.i_{1} \in\{1, \cdots, n\}, i_{2}, \cdots, i_{a} \in\{0,1, \cdots, n\}\right\}=T_{x}\left(\mathbb{R}^{d}\right) .
\end{aligned}\right.
$$

Then it is well-known that the heat equation $\frac{\partial}{\partial t}=E$ has the smooth heat kernel (=fundamental solution) $p(t, x, y)$. We are concerned with the diagonal short time asymptotics of it. In the case when $V_{0} \equiv 0$, under the assumption (1), they were obtained by Léandre [11] and Ben Arous [1]:

$$
p(i, x, x) \sim_{i}^{-N(x) / 2} \sum_{a=0}^{\infty} b_{a} i^{a} \quad \text { as } i \downarrow 0 .
$$

Here $N(x)$ is a positive integer defined in terms of $\left[\hat{V}_{a_{a}},\left[\hat{V}_{i_{a-1}},\left[\cdots,\left[\hat{V}_{i_{2}}, \hat{V}_{i_{1}}\right]\right.\right.\right.$ $\cdots](x), i_{1}, i_{2}, \cdots, i_{a} \in\{1, \cdots, n\}, 1 \leq a \leq \nu$ (more precisely, it is defined by (5.14)).

Communicated by S. Matsuura, March 26, 1987.

* Sugiyama Jogakuen University, Chikusa, INagoya 464, Japan. 
Previous to them, Bismut [2] discussed the not only diagonal but off diagonal short time asymptotics of $p(t, x, y)$ under the suitable conditions as an application of Wiener functional analysis; the splitting of the Wiener space and the use of an implicit function theorem. This approach by Bismut has been refined and expanded by Kusuoka [8] who introduced the notion of generalized Malliavin calculus. On the other hand, S. Watanabe [17], to solve this problem, introduced the notion of asymptotic expansions of generalized Wiener functionals. It should be noted that Léandre [11] further discussed the off diagonal short time asymptotics.

In this paper, following the way of S. Watanabe [17], we shall discuss the diagonal short time asymptotics in the general case (i.e. $V_{0} \neq 0$ ). This outline is as follows: Let $\left(W_{0}^{n}, P\right)$ be the $n$-dimensional Wiener space. For the operator $L$, we consider the following stochastic differential equation (SDE) of Stratonovich type on $\mathbb{R}^{d}$ :

$$
\left\{\begin{array}{l}
d X_{t}=\varepsilon \sum_{i=1}^{n} V_{i}\left(X_{t}\right) \circ d w_{t}^{i}+\varepsilon^{2} V_{0}\left(X_{t}\right) d t \\
X_{0}=x \in \mathbb{R}^{d}
\end{array}\right.
$$

where $\varepsilon>0$ and $w=\left(w_{t}^{i}\right) \in W_{0}^{n}$. Then the unique solution $X^{\varepsilon}(t, x)$ of this $\mathrm{SDE}$ is smooth in the Malliavin sense, and further, by virtue of the assumption (1), $X^{\varepsilon}(1, x)$ is non-degenerate in the Malliavin sense (cf. [4], [9], [16], [17]). Hence, for the Dirac delta-function $\delta_{x}\left(\in \mathcal{S}^{\prime}\left(\mathbb{R}^{d}\right)\right), \delta_{x}\left(X^{\mathbb{q}}(1, x)\right)$ is defined as a generalized Wiener functional and the probabilistic expression of $p\left(\varepsilon^{2}, x, x\right)$ is given:

$$
p\left(\varepsilon^{2}, x, x\right)=E\left[\delta_{x}\left(X^{\varepsilon}(1, x)\right)\right]
$$

(cf. [4], [16], [17]). First, for the integrand $\delta_{x}\left(X^{\mathrm{q}}(1, x)\right)$ in (2), we shall show the following asymptotic expansion (cf. [17]):

$$
\delta_{x}\left(X^{\varepsilon}(1, x)\right) \sim \varepsilon^{-N(x)} \sum_{a=0}^{\infty} \varepsilon^{a} \Theta_{a} \quad \text { as } \quad \varepsilon \downarrow 0
$$

provided that $\hat{V}_{0}(x)$ belongs to the linear subspace of $T_{x}\left(\mathbb{R}^{d}\right)$ spanned by $\hat{V}_{i}(x)$, $\left[\hat{V}_{j}, \hat{V}_{k}\right](x), i, j, k \in\{1, \cdots, n\}$ (cf. Theorem (5.31));

$$
\delta_{x}\left(X^{\varepsilon}(1, x)\right)=O\left(\varepsilon^{m}\right) \quad \text { as } \quad \varepsilon \downarrow 0 \quad \text { for any } m \geq 1
$$

provided that $\hat{V}_{0}(x)$ does not belong to that linear subspace (cf. Theorem (5.34)). Second, from (2), (3) $a,(3)_{b}$ and some observations, we shall show the short time asymptotic of $p(t, x, x)$ (cf. Theorem (6.8)): 
(4) $\left\{\begin{aligned} p(t, x, x) & \sim t^{-N(x) / 2} \sum_{a=0}^{\infty} t^{a} E\left[\Theta_{2 a}\right] \quad \text { as } t \downarrow 0, \quad \text { in the case (3) a } \\ =O\left(t^{m}\right) & \text { as } t \downarrow 0\end{aligned} \quad\right.$ for any $m \geq 1$, in the case (3)

Our argument seems to be simpler than Léandre's and Ben Arous', though it is based on the same idea as them.

As to another study of $p(t, x, y)$, there is the global estimate of it. This problem closely related to the above problem is investigated in many papers [3], [5], [10], [12], [13] etc. Among these, particularly, Kusuoka-Stroock [10] has obtained nice results by using the Malliavin calculus.

The organization of this paper is as follows: In $\S 1, \S 2$ and $\S 3$, we shall give some preliminaries for $\S 4, \S 5$ and $\S 6$. In particular, "a key" proposition in this paper will be presented in (3.9). In $\S 4$, with the aid of this proposition, we shall prove Proposition (4.4) which gives another look at Taylor's expansion of $X^{\varepsilon}(1, x)$ with respect to $\varepsilon$. In $\S 5$, by this proposition and by adopting Léandre's idea, the above $(3)_{a}$ and $(3)_{b}$ will be proved. In $\S 6$, the above (4) will be proved.

Warning. Throughout this paper, we freely use the notion, notations and the way of representations in [4], [16] and [17]. For details, refer to these papers.

\section{$\S$ H. Algebraic Preliminnaries}

Throughout this paper, let $n \geq 1$ be fixed. In this and the next section, we follow Yamato [18]. Set

$$
\begin{aligned}
& \mathbb{E}:=\{0,1, \cdots, n\}, \\
& \mathbb{E}_{a}:=\left\{\left(i_{1}, \cdots, i_{a}\right) ; i_{1}, \cdots, i_{a} \in \mathbb{W}\right\} \quad a \geq 1, \\
& \mathbb{E}(a):=\bigcup_{b=1}^{a} \mathbb{E}_{b}^{\prime} \quad 1 \leq a \leq \infty .
\end{aligned}
$$

For $I=\left(i_{1}, \cdots, i_{a}\right) \in \mathbb{E}(\infty)$, we introduce the following notations:

$$
\begin{aligned}
& |I|=\text { the length of } I:=a, \alpha(I):=\text { 䕓 }\left\{b \in\{1, \cdots, a\} ; i_{b}=0\right\}, \\
& \|I\|:=|I|+\alpha(I) .
\end{aligned}
$$

Set

$$
\begin{aligned}
& \hat{\mathbb{E}}(a):=\mathbb{E}(a) \backslash\{(0)\}, \quad \mathbb{E}((a)):=\{I \in \mathbb{E}(\infty) ;\|\mathbb{I}\| \leq a\} \subset \mathbb{E}(a), \\
& \hat{\mathbb{E}}((a)):=\mathbb{E}((a)) \backslash\{(0)\} .
\end{aligned}
$$

Let 


$$
\begin{aligned}
& \mathbb{R}(\mathbb{E}):=\text { the linear space with basis } \mathbb{E}, \\
& \mathbb{T}(\mathbb{E}):=\text { the tensor algebra generated by } \mathbb{R}(\mathbb{E}) \\
& =\mathbb{R} \oplus \mathbb{R}(\mathbb{E}) \oplus(\mathbb{R}(\mathbb{E}) \otimes \mathbb{R}(\mathbb{E})) \oplus \cdots, \\
& \mathbb{L}(\mathbb{E}):=\text { the } \mathbb{L} \text { ie subalgebra of } \mathbb{T}(\mathbb{E}) \text { generated by } \mathbb{E} .
\end{aligned}
$$

Here the bracket product in $\mathbb{T}(\mathbb{E})$ is defined by $[a, b]=a \otimes b-b \otimes a, a, b \in$ $\mathbb{T}(\mathbb{E})$. We define $\left[i_{1}, \cdots, i_{a}\right] \in \mathbb{E}(\mathbb{E})$ for $\left(i_{1}, \cdots, i_{a}\right) \in \mathbb{E}(\infty)$ by

$$
\left[i_{1}\right]:=i_{1}, \quad\left[i_{1}, \cdots, i_{a}\right]:=\left[\left[i_{1}, \cdots, i_{a-1}\right], i_{a}\right] \quad a \geq 2
$$

inductively. Each $\left[i_{1}, \cdots, i_{a}\right]$ is expressed as

$$
\left[i_{1}, \cdots, i_{a}\right]=\sum_{\left(j_{1}, \cdots, j_{b}\right) \in \mathbb{E}(\infty)} c_{i_{1}, \cdots, i_{b} j_{1}}^{j_{1}, \cdots} \cdots \otimes j_{b}
$$

and coefficients $c_{i_{1}, \cdots, i_{a}}^{j_{1}, \cdots, j_{b}}$ are uniquely determined by (1.1). Note that

$$
\left\{\begin{array}{lll}
\text { (i) } & c_{i}^{j}=\delta_{i}^{j} & \text { for } \quad i, j \in \mathbb{E}, \\
\text { (ii) } & c_{I}^{J}=0 & \text { if } \quad|\mathbb{I}| \neq|J|, \\
\text { (iii) } & c_{I}^{J}=0 & \text { if } \quad\|\mathbb{I}\| \neq\|J\| .
\end{array}\right.
$$

Set $r_{a}:=\operatorname{rank}\left[\left(c_{I}^{J}\right)_{I \in E_{a}, J \in E_{a}}\right] \quad a \geq 1$. Then $r_{a} \geq 1$ for any $a \geq 1$, and it is easy to see that

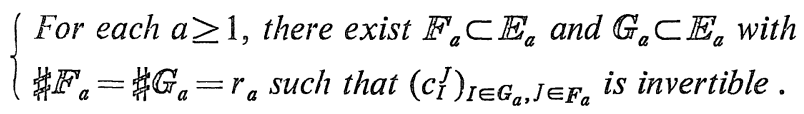

Clearly $\mathbb{F}_{1}=\mathbb{G}_{1}=\mathbb{E}$ by (1.2) (i). For each $1 \leq a \leq \infty$, set

$$
\begin{aligned}
& \mathbb{G}(a):=\bigcup_{b=1}^{a} \mathbb{G}_{b}, \quad \mathbb{F}(a):=\bigcup_{b=1}^{a} \mathbb{F}_{b}, \\
& \hat{\mathbb{G}}(a):=\mathbb{G}(a) \backslash\{(0)\}, \quad \hat{\mathbb{A}}(a):=\mathbb{F}(a) \backslash\{(0)\}, \\
& \mathbb{G}((a)):=\mathbb{G}(a) \cap \mathbb{E}((a)), \quad \mathbb{F}((a)):=\mathbb{F}(a) \cap \mathbb{E}((a)), \\
& \hat{\mathbb{G}}((a)):=\mathbb{G}((a)) \backslash\{(0)\}, \quad \hat{\mathbb{F}}((a)):=\mathbb{F}((a)) \backslash\{(0)\} .
\end{aligned}
$$

From (1.1), (1.2) and (1.3), we have the following, the proof of which is an elementary exercise of the linear algebra:

Proposition 1 .4. For each $a \geq 1$, the following holds:

(i) For a pair $(\mathbb{I}, \mathbb{J})=(\mathbb{E}(a), \mathbb{G}(a)),(\hat{\mathbb{E}}(a), \hat{\mathbb{G}}(a)),(\mathbb{E}((a)), \mathbb{G}((a)))$ and $(\hat{\mathbb{E}}((a))$, $\hat{G}((a)))$, respectively, $\{[J] ; J \in \mathbb{J}\}$ form a basis of the linear subspace (of $\mathbb{L}(\mathbb{E})$ ) spanned by $\{[I] ; I \in \mathbb{I}\}$.

(ii) Further, the linear subspace spanned by $\{[J] ; J \in \hat{G}(a)\}$ coincides with one spanned by $\{[(i, I)] ; i \in\{1, \cdots, n\}, I \in\{\phi\} \cup \mathbb{E}(a-1)\}$. Here $(i, I) \in \mathbb{E}(\infty)$ is 
defined by

$$
(i, I)=\left\{\begin{array}{l}
(i) \quad \text { if } \quad I=\phi \\
\left(i, i_{1}, \cdots, i_{b}\right) \quad \text { if } I=\left(i_{1}, \cdots, i_{b}\right) .
\end{array}\right.
$$

Let $\mathfrak{X}\left(\mathbb{R}^{r}\right)$ be the totality of $C^{\infty}$-vector fields on $\mathbb{R}^{r}$ with the bracket product $[X, Y]=X Y-Y X, X, Y \in \mathfrak{X}\left(\mathbb{R}^{r}\right) . \quad$ Let $X_{i} \in \mathfrak{X}\left(\mathbb{R}^{r}\right), i \in \mathbb{E}^{r}$ be given. For $\mathbb{I} \in$ $\mathbb{E}(\infty)$, define $X_{[I]} \in \mathbb{X}\left(\mathbb{R}^{r}\right)$ as follows:

$$
\begin{aligned}
& X_{\left[i_{1}\right]}:=X_{i_{1}}, \\
& X_{\left[i_{1}, \cdots, i_{a}\right]}:=\left[X_{\left[i_{1}, \cdots, i_{a-1}\right]}, X_{i_{a}}\right] \quad a \geq 2 .
\end{aligned}
$$

Also, we define a differential operator $X_{I}$ of order $|I|$ :

$$
X_{I}:=X_{i_{1}} \cdots X_{i_{a}} \quad \text { if } \quad I=\left(i_{1}, \cdots, i_{a}\right) .
$$

Then, as a corollary to (1.1) and (1.4), we have the following:

Corollary 1.5. (i) For each $I \in \mathbb{E}(\infty)$,

(ii) For each $a \geq 1$,

$$
X_{[I]}=\sum_{J \in \mathbb{E}(\infty)} c_{I}^{J} X_{J}
$$

$$
\begin{aligned}
& \text { Ł.1. }\left\{X_{[I]} ; \mathbb{I} \in \mathbb{E}(a)\right\}=\text { Һ.1. }\left\{X_{[I]} ; I \in \mathbb{G}(a)\right\} \text {, } \\
& \text { l.1. }\left\{X_{[I]} ; \mathbb{I} \in \hat{\mathbb{E}}(a)\right\}=\text { l.1. }\left\{X_{[r]} ; \mathbb{I} \in \hat{G}(a)\right\} \\
& =\text { l.1. }\left\{X_{[(i, I)]} ; i \in\{1, \cdots, n\}, \mathbb{I} \in\{\phi\} \cup \mathbb{E}(a-1)\right\} \text {, } \\
& \text { Ł.1. }\left\{X_{[I]} ; I \in \mathbb{E}((a))\right\}=\text { l.1. }\left\{X_{[I]} ; I \in \mathbb{G}((a))\right\} \text {, }
\end{aligned}
$$

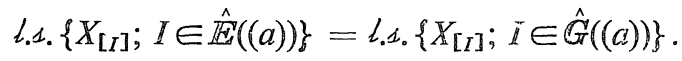

Here "l..." is an abbreviation for "linear span".

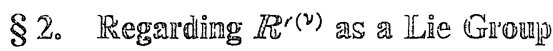

Throughout this section, we take an arbitrary $\nu \geq 1$ and fix it. Set

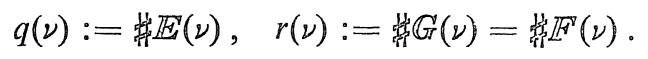

We identify the linear spaces (over $\mathbb{R}$ )

$$
\left\{\left(y^{I}\right)_{I \in E(\nu)} ; y^{I} \in \mathbb{R}^{1}, \mathbb{I} \in \mathbb{E}(\nu)\right\} \quad \text { and } \quad\left\{\left(u^{I}\right)_{I \in G(v)} ; u^{I} \in \mathbb{R}^{1}, \mathbb{I} \in \mathbb{G}(\nu)\right\}
$$

with $\mathbb{R}^{q(\nu)}$ and $\mathbb{R}^{r(\nu)}$, respectively. The coordinate systems on $\mathbb{R}^{q(\nu)}$ and $\mathbb{R}^{r(\nu)}$ are also denoted by $y^{I}, I \in \mathbb{E}(\nu)$ and $u^{I}, I \in \mathbb{G}(\nu)$, respectively. Define $Q_{i}=$ $Q_{i}^{(\nu)} \in \mathfrak{X}\left(\mathbb{R}^{q(\nu)}\right), i \in \mathbb{E}$ by 


$$
Q_{i}^{(\nu)}:=\frac{\partial}{\partial y^{i}}+\sum_{\substack{a+1 \leq \nu \\ j_{1}, \cdots, j_{a} \in \mathbb{E}}} y^{j_{1}, \cdots, j_{a}} \frac{\partial}{\partial y^{j_{1}, \cdots, j_{a}, i}} .
$$

For $I \in \mathbb{E}(\infty), Q_{[I]} \in \mathfrak{X}\left(\mathbb{R}^{q(v)}\right)$ is defined in the manner introduced in $\S 1$. Then, owing to Y. Yamato [18], we can state the following:

Proposition 2.1. (i) For $\left(i_{1}, \cdots, i_{a}\right) \in \mathbb{E}(\nu)$,

$$
Q_{\left[i_{1}, \cdots, i_{a}\right]}=\sum_{j_{1}, \cdots, j_{a} \in \mathbb{E}^{\prime}} c_{i_{1}, \cdots, i_{a}}^{j_{1}, \cdots, j_{a}}\left(\frac{\partial}{\partial y^{j_{1}, \cdots, j_{a}}}+\sum_{\substack{b+a \leq \nu \\ k_{1}, \cdots, k_{b} \in \mathbb{E}}} y^{k_{1}, \cdots, k_{b}} \frac{\partial}{\partial y^{k_{1}, \cdots, k_{b}, j_{1}, \cdots, j_{a}}}\right) .
$$

(ii) For $\left(i_{1}, \cdots, i_{a}\right) \in \mathbb{E}(\infty) \backslash(\nu), Q_{\left[i_{1}, \cdots, i_{a}\right]}=0$.

Let $\mathrm{g}=\mathrm{g}_{\nu}$ be the $\mathbb{L i e}$ subalgebra of $\mathfrak{X}\left(\mathbb{R}^{q(v)}\right)$ generated by $Q_{i}^{(\nu)}, i \in \mathbb{E}$. Then, from the above proposition, $\mathrm{g}$ is nilpotent of step $\nu$ and $\mathrm{g}=\ell_{. \iota} .\left\{Q_{[I]} ; I \in\right.$ $\mathbb{E}(\nu)\}$. Further, by (1.4)

$$
Q_{[I]}, I \in \mathbb{G}(\nu) \text { form a basis in } \mathrm{g} \text {. }
$$

As one more corollary to (2.1), we have the following: Let $\eta$ denote the coordinate system on $\mathbb{R}^{q(\nu)}$, i.e., $\eta^{J}\left(\left(y^{I}\right)_{I \in \mathbb{E}(\nu)}\right):=y^{J}, J \in \mathbb{E}(\nu)$. Then

Corollary 2.3. For $a \geq 1$ and $J \in \mathbb{E}(\nu)$,

$$
\begin{aligned}
& Q_{\left[I_{a}\right]} \cdots Q_{\left[I_{1}\right]} \eta^{J}
\end{aligned}
$$

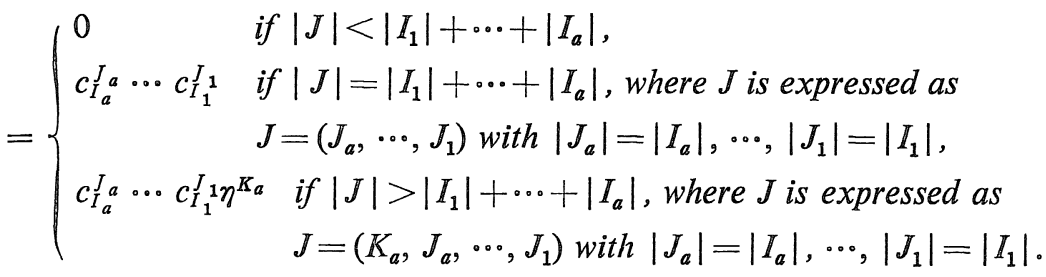

We denote by $\operatorname{Exp}(t Q)$ the integral curve of a complete vector field $Q$ $\left(\in \mathfrak{X}\left(\mathbb{R}^{q(\nu)}\right)\right)$. That is, for each $y=\left(y^{I}\right)_{I \in E(v)} \in \mathbb{R}^{q(\nu)}, \operatorname{Exp}(t Q)(y)$ is the unique solution of

$$
\left\{\begin{array}{l}
y_{t}=\left(y_{t}^{J}\right)_{J \in E(\nu)} \\
\frac{d}{d t} y_{t}^{J}=Q^{J}\left(y_{t}\right) \quad J \in \mathbb{E}(\nu) \\
y_{0}=y
\end{array}\right.
$$

where $Q^{J}, J \in \mathbb{E}(\nu)$ stand for the components of $Q$. The following is a consequence of (2.2) and (2.3): 
Corollary 2.4. If $Q \in \mathrm{g}$, then, for each $J \in \mathbb{E}(\nu)$ and $y=\left(y^{I}\right)_{I \in E(\nu)} \in \mathbb{R}^{q(\nu)}$,

$$
\operatorname{Exp}(t Q)(y)^{J}=\sum_{a=0}^{\nu} \frac{t^{a}}{a !}\left(Q^{a} \eta^{J}\right)(y)
$$

In particular,

$$
\operatorname{Exp}(Q)(y)^{J}=\sum_{a=0}^{\nu} \frac{1}{a !}\left(Q^{a} \eta^{J}\right)(y), \quad J \in \mathbb{E}(\nu)
$$

We define $\Phi=\Phi_{\nu} \in \mathbb{C}^{\infty}\left(\mathbb{R}^{r(\nu)} \times \mathbb{R}^{q(\nu)}, \mathbb{R}^{q(\nu)}\right)$ and $\varphi=\varphi_{\nu} \in C^{\infty}\left(\mathbb{R}^{r(\nu)}, \mathbb{R}^{q(\nu)}\right)$ as follows:

$$
\begin{aligned}
& \Phi_{\nu}(u, y):=\operatorname{Exp}\left(\sum_{I \in G(\nu)} u^{I} Q_{[I]}\right)(y) \\
& \varphi_{\nu}(u):=\bar{\Phi}_{\nu}(u, 0)=\operatorname{Exp}\left(\sum_{I \in G(\nu)} u^{I} Q_{[I]}\right)(0)
\end{aligned}
$$

Then $\Phi\left(u,{ }^{\circ}\right)$ is a diffeomorphism on $\mathbb{R}^{q(\nu)}$ for each $u \in \mathbb{R}^{r(v)}$, and particularly, $\Phi(0, \circ)$ is the identity mapping. By the Campbell-Hausdorff formula, for $u, v \in \mathbb{R}^{r(\nu)}$ we define a product $u \times v \in \mathbb{R}^{r(\nu)}$ so thai $\bar{\Phi}(u \times v, \circ)=\Phi(u, \bar{Q}(v, \circ))$ holds. With this multiplication, $\mathbb{R}^{r(\nu)}$ can be regarded as a Lie group with 0 as its identity. Let $\mathfrak{h}=\mathfrak{h}$, denote the right invariant Lie algebra of $\mathbb{R}^{r(\nu)}$ and let $\mathbb{R}_{i}=\mathbb{R}_{i}^{(\nu)} \in \mathfrak{h}_{\nu}$ be such that $\mathbb{R}_{i}(0)=\left(\frac{\partial}{\partial u^{i}}\right)_{0}, i \in \mathbb{2}$. Then $\mathrm{g}$ is isomorphic to $\mathfrak{G}$ under the correspondence: $Q_{i} \leftrightarrow \mathbb{R}_{i}$. Furthermore, if $\mathbb{R} \in \mathfrak{G}$ is an element corresponding to $Q \in \mathrm{g}$, then it holds that

$$
R(f \circ \varphi)=(Q f) \circ \varphi \quad f \in \mathbb{C}^{\infty}\left(\mathbb{R}^{q(\nu)}\right) .
$$

Note that for each $\mathbb{J} \in \mathbb{G}(\nu), R_{[J]} \in \mathfrak{h}$ is expressed as

$$
R_{[J]}=\left.\sum_{I \in G(v)} \frac{\partial}{\partial v^{J}}(v \times u)^{I}\right|_{v=0} \frac{\partial}{\partial u^{I}} .
$$

Also, the following holds: For $\lambda \neq 0$, we define an isomorphism $T_{(\nu)}^{(\lambda)}: \mathbb{R}^{r(\nu)} \rightarrow$ $\mathbb{R}^{r(\nu)}$ by

$$
T_{(\nu)}^{((\lambda))}\left((u)_{I \in G(\nu)}^{I}\right):=\left(\lambda\|\mu\| u^{I}\right)_{I \in G(\gamma)} .
$$

Then, for any $J \in \mathbb{G}(\nu)$

$$
\left(T_{(\nu)}^{(\lambda))}\right)_{*} R_{[J]}=\lambda\|J\| R_{[J]}
$$

Because $Q_{[J]}$ has the same property: For each $J \in \mathbb{E}(\nu)$

$$
\left(S_{(\nu)}^{(\lambda))}\right)_{*} Q_{[J]}=\lambda\|J\| Q_{[J]}
$$


where $S_{(\nu)}^{((\lambda))} \in \operatorname{Hom}\left(\mathbb{R}^{q(\nu)}, \mathbb{R}^{q(\nu)}\right)$ is defined similarly to $T_{(\nu)}^{((\lambda))}$.

To conclude this section, we make some remarks: (i) As a corollary to (2.4), we have that for each $K \in \mathbb{E}(\nu)$

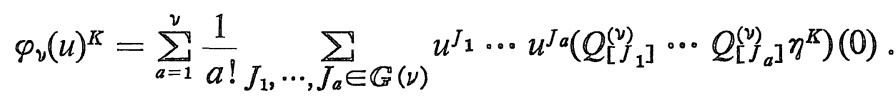

(ii) From (2.8) and (2.9), the following is derived by the same way as in Lemma (A.5) of Kusuoka-Stroock [10]:

$$
\varphi_{v} \text { is one-to-one . }
$$

(iii) For $\nu^{\prime} \geq \nu \geq 1$, define $\Pi \eta_{\nu}^{\nu^{\prime}} \in \operatorname{Hom}\left(\mathbb{R}^{q\left(\nu^{\prime}\right)}, \mathbb{R}^{q(\nu)}\right)$ and $P_{\nu}^{\nu^{\prime}} \in \mathbb{H o m}\left(\mathbb{R}^{r\left(\nu^{\prime}\right)}, \mathbb{R}^{r(\nu)}\right)$ as follows:

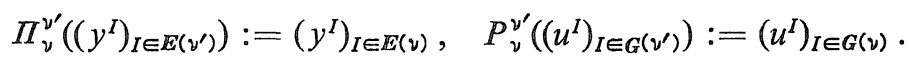

Then it holds that

$$
\Pi_{\nu}^{\nu^{\prime}} \circ \varphi_{v^{\prime}}=\varphi_{\nu} \circ \mathbb{P}_{\nu}^{\nu^{\prime}}
$$

(iv) Let $R_{[J]}^{(\nu) I}, \mathbb{I} \in \mathbb{G}(\nu)$ denote the components of $R_{[J]}^{(\nu)} \in \mathfrak{h}_{\nu}$. Then

$$
\inf \left\{\sum_{i=1}^{n} \sum_{K \in\{\phi\} \cup \mathbb{E}(\nu-1)}\left(\sum_{I \in \hat{\mathbb{G}}(\nu)} R_{[i, K)]}^{(\nu) I}(0) l^{I}\right)^{2} ; \sum_{I \in \hat{G}(\nu)}\left(l^{I}\right)^{2}=1\right\}>0 .
$$

Here we shall show (2.12) only: Suppose that for any $i \in\{1, \cdots, n\}$ and $K \in\{\phi\} \cup \mathbb{E}(\nu-1)$

$$
\sum_{I \in \hat{\mathbb{G}}(\nu)} \mathbb{R}_{[(i, K)]}^{(\nu) I}(0) l^{I}=0
$$

By (1.5) (ii), this implies that for any $J \in \hat{G}(\nu)$

$$
\sum_{I \in \hat{G}(\nu)} R_{[J]}^{(\nu) I}(0) l^{I}=0
$$

Since, by (2.6), $R_{[J]}^{(\nu) I}(0)=\left.\frac{\partial}{\partial v^{J}}(v \times 0)^{I}\right|_{v=0}=\delta_{J}^{I}, I, J \in G(\nu)$, this implies that $l^{J}=0$ for $J \in \hat{G}(\nu)$. Thus (2.12) follows immediately.

\section{§3. The Comtinnurus Process $\mathbb{U}_{t}^{(\nu)}$ on $\mathbb{R}^{r(\nu)}$}

Let $\left(W=W_{0}^{n}, P\right)$ be the $n$-dimensional Wiener space. Then a generic element $w=\left(w_{t}^{i}\right)$ of $W$ is clearly a realization of an $n$-dimensional Brownian motion starting at $0 \in \mathbb{R}^{n}$ under the measure $P$. Define the multiple Wiener integrals $w_{t}^{I}, \mathbb{I} \in \mathbb{E}(\infty)$ by 


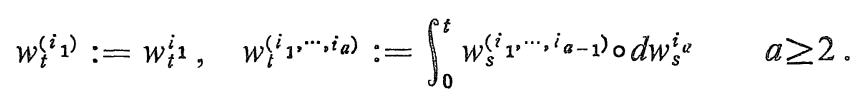

Here and hereafter, we set $w_{t}^{0}:=t$ for convenience. Then, for each $\nu \geq 1$, the following holds:

Proposition 3.1. (Y. Yamato [18]). The continuous process $\left[\left(w_{t}^{I}\right)_{I \in E(v)}\right.$; $t \geq 0]$ on $\mathbb{R}^{q(v)}$ is the unique solution of

$$
\left\{\begin{array}{l}
d Y_{t}=\sum_{i \in \mathbb{E}} Q_{i}^{(\nu)}\left(Y_{t}\right) \circ d w_{t}^{i} \\
Y_{0}=0 \in \mathbb{R}^{q(\nu)} .
\end{array}\right.
$$

The proof of (3.1) is obvious from the definition of $Q_{i}^{(\nu)}, i \in \mathbb{E}$.

Let $\nu \geq 1$ be fixed arbitrarily. We consider the following stochastic differential equation $(\mathrm{SDE})$ on $\mathbb{R}^{r(v)}$ :

$$
\left\{\begin{array}{l}
d U_{t}=\sum_{i \in \mathbb{E}} R_{i}^{(\nu)}\left(U_{i}\right) \circ d w_{t}^{i} \\
U_{0}=0 \in \mathbb{R}^{r(\nu)}
\end{array}\right.
$$

We denote by $U_{t}^{(\nu)}$ the unique solution of this $\mathrm{SDE}$. Then $U_{t}^{(\nu)} \in \mathbb{D}^{\infty}\left(\mathbb{R}^{r(\nu)}\right)$ for each $t \geq 0$. Let $Y_{t}$ and $Z_{t}$ be the unique solutions of the following SDE's on $\mathbb{R}^{r(\nu)} \otimes \mathbb{R}^{r(\nu)}$, respectively:

$$
\begin{aligned}
& \left\{\begin{array}{l}
d Y_{i}=\sum_{i \in \mathbb{E}} \partial R_{i}^{(\nu)}\left(U_{t}^{(\nu)}\right) Y_{t} \circ d w_{t}^{i} \\
Y_{0}=\left(\delta_{J}^{I}\right)_{I, J \in G(v)}
\end{array}\right. \\
& \left\{\begin{array}{l}
d Z_{t}=-\sum_{i \in \mathbb{E}} Z_{t} \partial R_{i}^{(\nu)}\left(U_{i}^{(\nu)}\right) \circ d w_{t}^{i} \\
Z_{0}=\left(\delta_{J}^{I}\right)_{I, J \in G}(\nu) .
\end{array}\right.
\end{aligned}
$$

Then $Z_{t} Y_{t}=\left(\delta_{J}^{I}\right)_{I, J \in G(\nu)}$. Further the following is well-known (cf. [4], [9], [16]): Let $\sigma_{(v)}=\left(\sigma_{v}^{I J}\right)_{I, J \in G(v)}$ be the Malliavin covariance of $U_{1}^{(v)}$ :

$$
\sigma_{\nu}^{I J}:=\left\langle D U_{1}^{(\nu) I}, D U_{1}^{(\nu)^{J}}\right\rangle \quad \mathbb{I}, \mathbb{J} \in \mathbb{G}_{r}^{(\nu)} .
$$

Set $\tau_{(v)}=\left(\tau_{v}^{I J}\right)_{I, J \in G(v)}$ :

$$
\tau_{\nu}^{I J}:=\sum_{i=1}^{n} \int_{0}^{1}\left(Z_{s} R_{i}^{(\nu)}\left(U_{s}^{(\nu)}\right)\right)^{I}\left(Z_{s} R_{i}^{(\nu)}\left(U_{s}^{(\nu)}\right)\right)^{J} d s \quad I, J \in G(\nu) .
$$

Then $\sigma_{(v)}=Y_{1} \tau_{(v)}\left(Y_{1}\right)^{*}$. Note that for any $u, v \in \mathbb{R}^{r(v)}$

$$
(u \times v)^{i}=u^{i}+v^{i} \quad i \in \mathbb{E} .
$$

This is easily verified by viewing the Campbell-Hausdorff series: 


$$
\begin{aligned}
\sum_{I \in G(\nu)}(u \times v)^{I} Q_{[I]}^{(\nu)}= & \sum_{I \in G(\nu)}\left(v^{I}+u^{I}\right) Q_{[I]}^{(\nu)} \\
& +\frac{1}{2}\left[\sum_{I \in \mathbb{G}(\nu)} v^{I} Q_{[I]}^{(\nu)}, \sum_{I \in \mathbb{G}(\nu)} u^{I} Q_{[I]}^{(\nu)}\right]+\cdots
\end{aligned}
$$

Combining (3.5) with (2.6), we see that $R_{i}^{(y)^{j}}(\circ)=\delta_{i}^{j}, i, j \in \mathbb{E}$. Hence, in view of (3.2) and (3.3)

$$
U_{t}^{(\nu)^{j}}=w_{t}^{j}, \quad Y_{t}^{j J}=\delta_{J}^{j} \quad j \in \mathbb{E}, J \in \mathbb{G}(\nu) .
$$

Since $Z_{t} Y_{t}=Y_{\xi} Z_{t}=\left(\delta_{J}^{I}\right)_{I_{0} J \in G(v)}$, we further see that $Z_{t}^{j J}=\delta_{J}^{j}, j \in \mathbb{E}, J \in G(\nu)$. Thus, if we set

$$
\begin{array}{ll}
\hat{Y}_{t}:=\left(Y_{t}^{I J}\right)_{I, J \in \hat{G}}(\nu), & \hat{Z}_{t}:=\left(Z_{t}^{I J}\right)_{I, J \in \hat{G}(\nu),}, \\
\hat{\sigma}_{(\nu)}:=\left(\sigma_{\nu}^{I J}\right)_{I_{0} J \in \hat{G}(\nu)}, \quad \hat{\tau}_{(\nu)}:=\left(\tau_{\nu}^{I J}\right)_{I, J \in \hat{G}(\nu)},
\end{array}
$$

then we have

$$
\left\{\begin{array}{l}
Y_{q}=\left[\begin{array}{ll}
1 & 0 \\
* & \hat{Y}_{t}
\end{array}\right], \quad Z_{t}=\left[\begin{array}{cc}
1 & 0 \\
* * & \hat{Z}_{t}
\end{array}\right], \\
\sigma_{(v)}=\left[\begin{array}{ll}
0 & 0 \\
0 & \hat{\sigma}_{(v)}
\end{array}\right], \quad \tau_{(v)}=\left[\begin{array}{ll}
0 & 0 \\
0 & \hat{\tau}_{(v)}
\end{array}\right], \\
\hat{Z}_{t} \hat{Y}_{t}=\left(\delta_{J}^{I}\right)_{I, J \in \hat{G}(v)}, \quad \hat{\sigma}_{(v)}=\hat{Y}_{1} \hat{\tau}_{(v)}\left(\hat{Y}_{1}\right)^{*} .
\end{array}\right.
$$

Now, owing to Kusuoka-Stroock [9], we can state the following: Set

Then

$$
\hat{\pi}_{(\nu)}:=\inf \left\{\left\langle\hat{\sigma}_{(\nu)} \hat{l}, \hat{l}\right\rangle ; \hat{l}=\left(l^{I}\right)_{I \in \hat{G}(\nu)} \quad \text { such as } \sum_{I \in \hat{G}(\nu)}\left(l^{I}\right)^{2}=1\right\} \text { 。 }
$$

Proposition 3.8. It holds that $\hat{\pi}_{(v)}>0$ a.s. $(P)$ and

$$
\left(\hat{\pi}_{(v)}\right)^{-1} \in L_{\infty-}:=\bigcap_{p>1} L_{p} .
$$

Proof. First of all, set

$$
\hat{\rho}_{(\nu)}:=\inf \left\{\langle\hat{\tau}(\nu) \hat{l}, \hat{l}\rangle ; \hat{l}=\left(l^{I}\right)_{I \in \hat{G}(\nu)} \text { such as } \sum_{I \in \hat{G}(\nu)}\left(l^{I}\right)^{2}=1\right\} .
$$

It is sufficient to show (3.8) for $\hat{\rho}_{(v)}$. For, we observe by (3.7) that for $\hat{l}=\left(l^{I}\right)_{I \in G(\nu)}$ such as $\sum_{I \in \hat{G}(\nu)}\left(l^{I}\right)^{2}=1$

$$
\begin{aligned}
\left\langle\hat{\sigma}_{(v)} \hat{l}, \hat{l}\right\rangle & =\left\langle\hat{Y}_{1} \hat{\tau}_{(v)}\left(\hat{Y}_{1}\right) * \hat{l}, \hat{l}\right\rangle=\left\langle\hat{\tau}_{(v)}\left(\hat{Y}_{1}\right)^{*} \hat{l}_{9}\left(\hat{Y}_{1}\right)^{*} \hat{l}\right\rangle \\
& =\left|\left(\hat{Y}_{1}\right) * \hat{l}\right|^{2}\left\langle\hat{\tau}_{(v)} \frac{\left(\hat{Y}_{1}\right)^{*} \hat{l}}{\left|\left(\hat{Y}_{1}\right)^{*} \hat{l}\right|}, \frac{\left(\hat{Y}_{1}\right)^{*} \hat{l}}{\left|\left(\hat{Y}_{1}\right)^{*} \hat{l}\right|}\right\rangle
\end{aligned}
$$




$$
\geq\left|\left(\hat{\Psi}_{1}\right) * \hat{l}\right|^{2} \hat{\rho}_{(v)} \geq\left\|\left(\hat{\mathbb{Z}}_{1}\right)^{*}\right\|^{-2} \hat{\rho}_{(v)}
$$

Noticing that $\left\|\left(\hat{Z}_{1}\right)^{*}\right\| \in L_{\infty}$ by (3.4), we see (3.8) for $\hat{\pi}_{(y)}$ at once.

Now we shall prove (3.8) for $\hat{\rho}_{(v)}$. In view of (3.2) and (3.4), we observe by Itô's formula that

$$
\begin{aligned}
& Z_{t} R_{i}^{(\nu)}\left(U_{t}^{(\nu)}\right) \\
& \left.\quad=R_{i}^{(\nu)}(0)+\sum_{i_{1} \in \mathbb{E}}\left[R_{i_{1}}^{(\nu)}, R_{i}^{(\nu)}\right](0) w_{t}^{i}\right]+\sum_{i_{1}, i_{2} \in \mathbb{E}}\left[R_{i_{1}}^{(\nu)},\left[R_{i_{2}}^{(\nu)}, R_{i}^{(\nu)}\right]\right](0) w_{i}^{i_{1}, i_{2}} \\
& \quad+\cdots+\sum_{i_{1}, \cdots, i_{\nu-1} \in \mathbb{E}}\left[R_{i_{1}}^{(\nu)},\left[R_{i_{2}}^{(\nu)}, \cdots,\left[\mathbb{R}_{i_{\nu-1}}^{(\nu)}, R_{i}^{(\nu)}\right] \circ\right](0) w_{i}^{i_{1}, \cdots, i_{\nu-1}}\right.
\end{aligned}
$$

where we have used the fact: $\left[\mathbb{R}_{j_{1}}^{(\nu)},\left[R_{j_{2}}^{(\nu)}, \cdots,\left[R_{j_{a-1}}^{(\nu)}, \mathbb{R}_{j_{a}}^{(\nu)}\right] \cdots\right]=0\right.$ for any $a \geq \nu+1$. Since, in general, it holds that

$$
\left[R_{i_{1}}^{(\nu)},\left[R_{i_{2}}^{(\nu)}, \cdots,\left[R_{i_{a}}^{(\nu)}, R_{i}^{(\nu)}\right] \cdots\right]=(-1)^{a} R_{\left[i_{0} i_{a}, \cdots, i_{1}\right]}^{(\nu)},\right.
$$

the above is equal to

$$
\left.\sum_{I \in\{\phi\} \cup \mathbb{E}(\nu-1)}(-1)^{|I|} \mathbb{R}_{[\{i, \nu}^{(\nu)}\right](0) w_{t}^{I} .
$$

Here, for convenience sake, we set that $|\phi|:=0, w_{s}^{\phi}:=1$ and

$$
\check{I}:=\left\{\begin{array}{l}
\phi \quad \text { if } \quad \mathbb{I}=\dot{\phi} \\
\left(i_{a}, \ldots \circ, i_{1}\right) \quad \text { if } \quad I=\left(i_{1}, \ldots, i_{a}\right) .
\end{array}\right.
$$

Hence, recalling (3.7) and the definition of $\tau(\vartheta)$, we see that for $\hat{l}=\left(l^{I}\right)_{I \in \hat{G}(\vartheta)}$

$$
\left\langle\hat{\tau}_{(\nu)} \hat{l}, \hat{l}\right\rangle=\sum_{i=1}^{n} \int_{0}^{1}\left(\sum_{K \in\{\phi\} \cup \mathbb{E}(\nu-1)} \sum_{I \in \hat{\mathbb{G}}(\nu)}(-1)^{|K|} R_{[(i, \underline{K})]}^{(\nu)}(0) l^{I} w_{s}^{K}\right)^{2} d s .
$$

Thus, setting

$$
\mathcal{V}_{\nu}(\hat{l}):=\sum_{i=1}^{n} \sum_{K \in\{\phi\} \cup \mathbb{E}(\nu-1)}\left(\sum_{I \in \hat{G}(\nu)} R_{[i, K)]}^{(\nu)}(0) l^{I}\right)^{2}, \quad \hat{l}=\left(l^{I}\right)_{I \in \hat{G}(\nu)}
$$

we have

$$
\begin{aligned}
\hat{\rho}_{(\nu)} \geq & \frac{1}{n} \inf \left\{C V_{\nu}(\hat{l}) ; \sum_{r \in \hat{\mathbb{G}}(\nu)}\left(l^{I}\right)^{2}=1\right\} \\
& \times \inf \left\{\int_{0}^{1}\left(\sum_{K \in\{\phi\} \cup \mathbb{E}(\nu-1)} b_{K} w_{s}^{K}\right)^{2} d s ; \sum_{K \in\{\phi\} \cup \mathbb{E}(\nu-1)}\left(b_{K}\right)^{2}=1\right\} .
\end{aligned}
$$

Consequently, from Theorem (A.6) in Kusuoka-Stroock [9] and (2.12), (3.8) for $\hat{\rho}_{(y)}$ follows. //

The following proposition is a key in this paper, though its proof is easy. 
Proposition 3.9. For each $I \in \mathbb{E}(\nu)$,

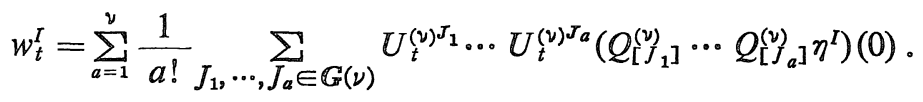

Proof. Let $f \in C^{\infty}\left(\mathbb{R}^{q(\nu)}\right)$. By (3.2) and (2.5), we observe

$$
d f\left(\varphi_{\nu}\left(U_{t}^{(\nu)}\right)\right)=\sum_{i \in \mathbb{E}} R_{i}^{(\nu)}\left(f \circ \varphi_{\nu}\right)\left(U_{t}^{(\nu)}\right) \circ d w_{t}^{i}=\sum_{i \in \mathbb{E}}\left(Q_{i}^{(\nu)} f\right)\left(\varphi_{\nu}\left(U_{t}^{(\nu)}\right)\right) \circ d w_{t}^{i} .
$$

By (3.1), this implies

$$
\left(w_{t}^{I}\right)_{I \in E(v)}=\varphi_{\nu}\left(U_{t}^{(\nu)}\right),
$$

from which and (2.9), (3.9) follows at once. //

Let $\nu^{\prime} \geq \nu \geq 1$. Recalling (2.11), we can state a relation between $U_{t}^{\left(\nu^{\prime}\right)}$ and $U_{t}^{(\nu)}$.

Proposition 3.11. $\quad P_{\nu}^{\nu^{\prime}} U_{t}^{\left(\nu^{\prime}\right)}=U_{t}^{(\nu)} \quad t \geq 0$.

Proof. By (2.11), we observe

$$
\Pi_{\nu}^{\nu^{\prime}}\left(\varphi_{\nu}\left(U_{t}^{\left(\nu^{\prime}\right)}\right)\right)=\varphi_{\nu}\left(P_{\nu}^{\nu^{\prime}} U_{t}^{\left(\nu^{\prime}\right)}\right)
$$

By (3.10), this implies that $\varphi_{\nu}\left(U_{t}^{(\nu)}\right)=\varphi_{\nu}\left(P_{\nu}^{\nu^{\prime}} U_{t}^{\left(\nu^{\prime}\right)}\right)$. Hence, (3.11) follows from (2.10). //

By virtue of (3.11), we can define a continuous process $\left[U_{t}^{(\infty)} ; t \geq 0\right]$ on $\mathbb{R}^{\infty} \simeq\left\{\left(u^{I}\right)_{I \in G(\infty)} ; u^{I} \in \mathbb{R}^{1}, I \in \mathbb{G}(\infty)\right\}$ so that $P_{\nu}^{\infty} U_{t}^{(\infty)}=U_{t}^{(\nu)}$ for any $\nu \geq 1$. Let $U_{t}^{I}, I \in G(\infty)$ be the components of $U_{t}^{(\infty)}$. For $\lambda \neq 0$, an isomorphism $T^{((\lambda))}: \mathbb{R}^{\infty} \rightarrow \mathbb{R}^{\infty}$ is defined by

$$
T^{((\lambda))}\left(\left(u^{I}\right)_{I \in G(\infty))}:=\left(\lambda^{\|I\|} u^{I}\right)_{I \in G(\infty)} .\right.
$$

Then

Proposition 3.12. (i) $\left[T^{((\lambda))} U_{t}^{(\infty)} ; t \geq 0\right]$ is equivalent in law to $\left[U_{\lambda^{2} t}^{(\infty)} ; t \geq 0\right]$. (ii) For any $\mathbb{I} \in \mathbb{G}(\infty), U_{t}^{I}(-w)=(-1)^{\|I\|} U_{t}^{I}(w)$.

Proof. Let $\nu \geq 1$ be fixed arbitrarily. Let $V_{t}^{((\lambda))}$ denote the unique solution of

$$
\left\{\begin{array}{l}
d V_{t}=\lambda \sum_{i=1}^{n} R_{i}^{(\nu)}\left(V_{t}\right) \circ d w_{t}^{i}+\lambda^{2} R_{0}^{(\nu)}\left(V_{t}\right) d t \\
V_{0}=0 \in \mathbb{R}^{r(\nu)} .
\end{array}\right.
$$

Then, from (2.7) and (3.2), it is easy to see that

$$
V_{t}^{((\lambda))}=T_{(\nu)}^{((\lambda))} U_{t}^{(\nu)} \quad t \geq 0
$$


On the other hand, from the scaling property of $\left(w_{t}^{i}\right)_{i \in\{1, \cdots, n\}}$, it is clear that $\left[V_{t / \lambda^{2}}^{((\lambda))} ; t \geq 0\right]$ is equivalent in law to $\left[U_{t}^{(\nu)} ; t \geq 0\right]$. Hence, combining this and (3.13), we have the assertion (i). For (ii), if we take $\lambda=-1$, then $V_{t}^{((-1))}(w)=$ $U_{t}^{(\nu)}(-w)$, and thus, this, together with (3.13), implies the assertion (ii). //

We end this section with the following remark: Set

$$
m_{\nu, \kappa}:=\max _{\left|u^{u}\right| \leq^{\kappa}}\left|\frac{1}{2} \sum_{i=1}^{n} \partial R_{i}^{(\nu)}(u) \circ R_{i}^{(\nu)}(u)+R_{0}^{(\nu)}(u)\right|+\max _{\left|{ }^{u}\right| \leqq k} \sum_{i=1}^{n}\left|R_{i}^{(\nu)}(u)\right|^{2} 。
$$

Note that $m_{\nu, \kappa}>0$, since $R_{i}^{(\nu) I}(0)=\delta_{i}^{I}, i \in \mathbb{E}, \mathbb{I} \in \mathbb{G}(\nu)$. Then, by the standard procedure due to Stroock-Varadahn [15], we can obtain that for any $0<t \leq$ $\frac{1}{2} \frac{\kappa}{r(\nu) m_{\nu, \kappa}}$

$$
P\left(\max _{0 \leq s \leq t}\left|U_{s}^{(\nu)}\right| \geq \kappa\right) \leq 2 r(\nu) \exp \left(-\frac{1}{8} \frac{\kappa^{2}}{r(\nu)^{2} m_{\nu, k}} \frac{1}{t}\right) .
$$

Thus, by putting this and (3.12) (i) together, the following estimate holds: For $t>0$ and $0<\varepsilon \leq\left(\frac{1}{2} \frac{r}{r(\nu) m_{\nu, \kappa} t}\right)^{1 / 2}$,

$$
P\left(\max _{0 \leq s \leq t}\left|T_{(\nu)}^{(()))} U_{s}^{(\nu)}\right| \geq \kappa\right) \leq 2 r(\nu) \exp \left(-\frac{1}{8} \frac{\kappa^{2}}{r(\nu)^{2} m_{\nu, \kappa}} \frac{1}{t} \frac{1}{\varepsilon^{2}}\right) .
$$

\section{§4. The Smooth (in the Mialliavin Semse)}

Wiener Fumctional $X^{\varepsilon}(\mathbb{1}, x)$

Let $V_{i} \in C_{b}^{\infty}\left(\mathbb{R}^{d}, \mathbb{R}^{d}\right), i \in \mathbb{E}$. Define $\hat{V}_{i} \in \mathfrak{x}\left(\mathbb{R}^{d}\right), i \in \mathbb{E}$ and a second order differential operator $L$ on $\mathbb{R}^{d}$ as follows:

$$
\begin{aligned}
& \hat{V}_{i}:=\sum_{j=1}^{d} V_{i}^{j} \frac{\partial}{\partial x^{j}} \quad i \in \mathbb{E}, \\
& L:=\frac{1}{2} \sum_{i=1}^{n} \hat{V}_{i}^{2}+\hat{V}_{0} .
\end{aligned}
$$

Let $\left(W=W_{0}^{n}, P\right)$ be, as before, the $n$-dimensional Wiener space. For $\varepsilon>0$, we consider the following SDE on $\mathbb{R}^{d}$ :

$$
\left\{\begin{array}{l}
d X_{t}=\varepsilon \sum_{i=1}^{n} V_{i}\left(X_{t}\right) \circ d w_{t}^{i}+\varepsilon^{2} V_{0}\left(X_{t}\right) d t \\
X_{0}=x \in \mathbb{R}^{d}
\end{array}\right.
$$

We denote by $X^{\varepsilon}(t, x)$ the unique solution of this SDE. Then, for each $t \geq 0$ and $x \in \mathbb{R}^{d}, X^{\varepsilon}(t, x) \in \mathbb{D}^{\infty}\left(\mathbb{R}^{d}\right)$. Further, the following is well-known as Taylor's 
expansion of $X^{\mathbf{q}}(t, x)$ with respect to $\varepsilon$ (cf. [9], [17]): Let $\zeta$ be the coordinate system on $\mathbb{R}^{d}$, i.e., $\zeta^{i}(x)=x^{i}$ for $i \in\{1, \cdots, d\}$. Then, for each $a \geq 1$

$$
\left\{\begin{array}{c}
X^{\varepsilon}(t, x)=x+\sum_{I \in \mathbb{E}(a)}\left(\hat{V}_{I} \zeta\right)(x) \varepsilon^{\|I\|} w_{t}^{I} \\
+\sum_{\left(i_{1}, \cdots, i_{a+1}\right) \in \mathbb{E}_{a+1}} \varepsilon^{\left\|\left(i_{1}, \cdots, i_{a+1}\right)\right\|} \int_{0}^{t} \circ d w_{t_{a+1}}^{i_{a+1}} \int_{0}^{t_{a+1}} \circ d w_{t_{a}}^{i_{a} \cdots} \\
\cdots \int_{0}^{t_{2}}\left(\hat{V}_{\left(i_{1}, \cdots, i_{a+1}\right.} \zeta\right)\left(X^{\mathcal{q}}\left(t_{1}, x\right)\right) \circ d w_{t_{1}^{1}}^{i_{1}} .
\end{array}\right.
$$

By using (3.9), we shall rewrite (4.2). For this, we introduce $F_{\nu}^{q}(t, x), R_{\nu}^{q}(t, x)$ $\in \mathbb{D}^{\infty}\left(\mathbb{R}^{d}\right), \nu \geq 1$ :

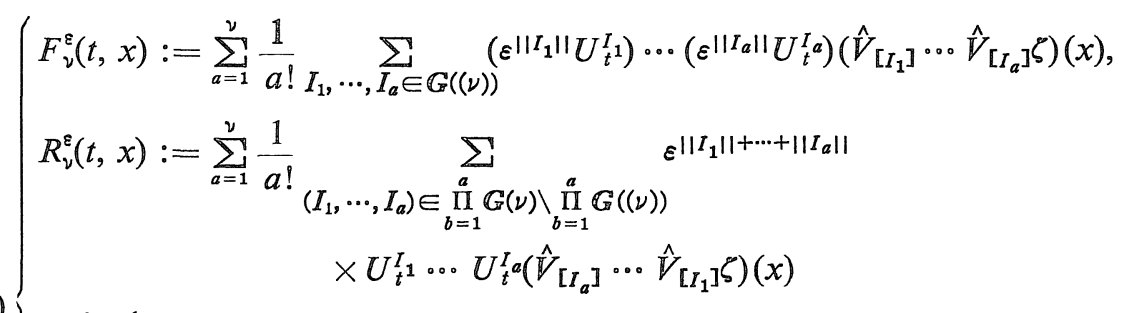

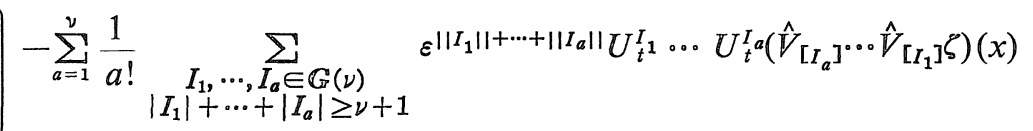

$$
\begin{aligned}
& +\sum_{\left(i_{1}, \cdots, i_{\nu+1}\right) \in \mathbb{E}_{\nu+1}} \varepsilon^{\left\|\left(i_{1}, \cdots, i_{y+1}\right)\right\|} \int_{0}^{t} \circ d w_{t_{\nu+1}}^{i_{\nu+1}} \int_{0}^{t_{\nu+1}} \circ d w_{t_{\nu}}^{i_{\nu}} \ldots \\
& \cdots \int_{0}^{t_{2}}\left(\hat{V}_{\left(i_{1}, \cdots, i_{v+1}\right.} \zeta\right)\left(X^{\varepsilon}\left(t_{1}, x\right)\right) \circ d w_{t_{1}}^{i_{1}} \text {. }
\end{aligned}
$$

Here recall that $U_{t}^{I}, I \in G(\infty)$ are the components of $U_{t}^{(\infty)}$ defined in $\S 3$. Then

Proposition 4.4. It holds that

$$
X^{\varepsilon}(t, x)=x+F_{\nu}^{\varepsilon}(t, x)+R_{\nu}^{\varepsilon}(t, x) .
$$

Proof. Let $\nu \geq 1$ be fixed arbitrarily. By virtue of (3.9), we observe that

$$
\begin{aligned}
& \sum_{J \in \mathbb{E}(\nu)}\left(\hat{V}_{I} \zeta\right)(x) \varepsilon^{\|I\|} W_{t}^{I}
\end{aligned}
$$

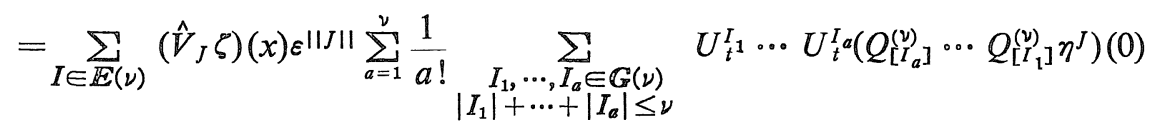

$$
\begin{aligned}
& =\sum_{a=1}^{\nu} \frac{1}{a !} \sum_{\substack{I_{1}, \cdots, I_{a} \in G(\nu) \\
\left|I_{1}\right|+\cdots+\left|I_{a}\right| \leq \nu}} U_{t^{1}}^{I_{1}} \cdots U_{t}^{I^{a}} \sum_{J \in \mathbb{E}(\nu)} \varepsilon^{\|J\|}\left(\hat{V}_{J} \zeta\right)(x)\left(Q_{\left[I_{a}\right]}^{(\nu)} \cdots Q_{\left[I_{1}\right]}^{(\nu)} \eta^{J}\right)(0) .
\end{aligned}
$$

Since, by (2.3) 


$$
\begin{aligned}
& \left(Q_{\left[I_{a}\right]}^{(\nu)} \cdots Q_{\left[I_{1}\right]}^{(\nu)} \eta^{J}\right)(0) \\
& =\left\{\begin{array}{lll}
0 & \text { if }|J| \neq\left|I_{1}\right|+\cdots+\left|I_{a}\right| \\
c_{I_{a}}^{J} \cdots & c_{I_{1}}^{J} & \text { if }|J|=\left|I_{1}\right|+\cdots+\left|I_{a}\right|, \text { where } J \text { is expressed as } \\
& J=\left(J_{a}, \cdots, J_{1}\right) \text { with }\left|J_{a}\right|=\left|I_{a}\right|, \cdots,\left|J_{1}\right|=\left|I_{1}\right|,
\end{array}\right.
\end{aligned}
$$

the above is equal to

$$
\begin{aligned}
& \sum_{a=1}^{\nu} \frac{1}{a !} \sum_{\substack{I_{1}, \cdots, I_{a} \in \mathbb{G}(\nu) \\
\left|I_{1}\right|+\cdots+\left|I_{a}\right| \leq \nu}} U_{t^{1}}^{I_{1} \cdots U_{t}^{I_{a}}} \\
& \times \sum_{\left|I_{1}\right|=\mid J_{1}, \cdots, \cdots, J_{a} \in \mathbb{E}(\nu)} \varepsilon^{\left\|\left(J_{a}, \cdots, J_{1}\right)\right\|}\left(\hat{V}_{\left(J_{a}, \cdots, J_{1}\right)} \zeta\right)(x) c_{I_{1}}^{J} \ldots J_{a} \mid c_{I_{a}}^{J}
\end{aligned}
$$

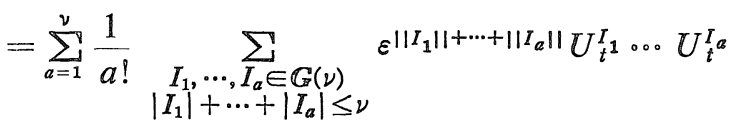

$$
\begin{aligned}
& \times \sum_{J_{1}, \cdots, J_{a} \in \mathbb{E}(\nu)}\left(\left(c_{I_{a}}^{J_{a}} \hat{V}_{J_{a}}\right) \cdots\left(c_{I_{1}}^{J} \hat{V}_{J_{1}}\right) \xi\right)(x)
\end{aligned}
$$

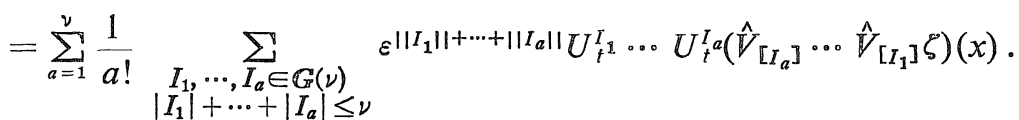

Here the last equality has come from (1.5) (i). Thus, putting (4.2), (4.3) and the above together, we obtain (4.4) immediately. //

For $I \in \mathbb{E}(\infty)$, let $V_{[I]}=\left(V_{[I]}^{i}\right)_{i \in\{1, \cdots, d\}} \in C_{b}^{\infty}\left(\mathbb{R}^{d}, \mathbb{R}^{d}\right)$ denote the components of $\hat{V}_{[I]} \in \mathfrak{X}\left(\mathbb{R}^{d}\right): \hat{V}_{[I]}=\sum_{j=1}^{d} V_{[I]}^{j} \frac{\partial}{\partial x^{j}} . \quad$ Define $\quad \mathbb{V}_{I_{1}, \cdots, I_{a}}=\left(\mathbb{V}_{I_{1}, \cdots, I_{a}}^{i j}\right)_{i, j \in\{1, \cdots, d\}} \in$ $C_{b}^{\infty}\left(\mathbb{R}^{d}, \mathbb{R}^{d} \otimes \mathbb{R}^{d}\right)$ for $I_{1}, \cdots, I_{a} \in \mathbb{E}(\infty)$ and $a \geq 1$ by

$$
\mathbb{V}_{I_{1}, \cdots, I_{a}}^{i j}:=\partial_{j}\left(\hat{V}_{\left[I_{1}\right]} \cdots \hat{V}_{\left[I_{a}\right]} \zeta^{i}\right) \quad i, j \in\{1, \cdots, d\} .
$$

For convenience, we set $\mathbb{V}_{\phi}:=I_{R^{d}}$ if $I=\phi$. Then we easily see that for $i \in\{1, \cdots, d\}$ and $I_{1}, \cdots, I_{a} \in \mathbb{E}(\infty)$

$$
\hat{V}_{\left[I_{1}\right]} \cdots \hat{V}_{\left[I_{a}\right]} \zeta^{i}=\sum_{j=1}^{d} V_{\left[I_{1}\right]}^{j} \nabla_{I_{2} \cdots, I_{a}}^{i j} \text { 。 }
$$

For each $\nu \geq 1$, we define $\alpha_{\nu}=\left(\alpha_{\nu}^{i I}\right)_{i \in\{1, \cdots, d\}, I \in G((\nu))} \in C_{b}^{\infty}\left(\mathbb{R}^{d}, \mathbb{R}^{d} \otimes \mathbb{R}^{r((\nu))}\right)$ and $M_{\nu}=\left(M_{\nu}^{i j}\right)_{i, j \in\{1, \cdots, d\}} \in C^{\infty}\left(\mathbb{R}^{d} \times \mathbb{R}^{r}((\nu)), \mathbb{R}^{d} \otimes \mathbb{R}^{d}\right)$ as follows:

$$
\begin{aligned}
& \alpha_{\nu}^{i I}(x):=V_{[I]}^{i}(x), \\
& M_{\nu}^{i j}\left(x,\left(u^{I}\right)_{I \in G((v)))}:=\delta_{j}^{i}+\sum_{a=1}^{\nu-1} \frac{1}{(a+1) !} \sum_{I_{1}, \cdots, I_{a} \in \mathbb{G}((\nu))} \mathbb{V}_{I_{1}, \cdots, I_{a}}^{i j}(x) u^{I_{1}} \ldots u^{I_{a}} 。\right.
\end{aligned}
$$

Here, as before, we identify 


$$
\left\{\left(u^{I}\right)_{I \in G((v))} ; u^{I} \in \mathbb{R}^{1}, \mathbb{I} \in \mathbb{G}((\nu))\right\}
$$

with $\mathbb{R}^{r((v))}=\mathbb{R}^{\mathbb{G}((v))}$. Set $F_{\nu}(x, u):=M_{\nu}(x, u) \alpha_{\nu}(x) u \in C^{\infty}\left(\mathbb{R}^{d} \times \mathbb{R}^{r((v))}, \mathbb{R}^{d}\right)$. Then, from (4.3) and (4.5), we have

Proposition 4.6. It holds that

$$
F_{\nu}^{\varepsilon}(t, x)=F_{\nu}\left(x, T_{((\nu))}^{((\varepsilon))} U_{t}^{((\nu))}\right) .
$$

Here $U_{t}^{((v))}:=\left(U_{t}^{I}\right)_{I \in G((v))}$ and $\mathbb{T}_{((\nu))}^{((\varepsilon))} \in \operatorname{Hom}\left(\mathbb{R}^{r((\nu))}, \mathbb{R}^{r((\nu))}\right)$ is defined by $T_{((\nu))}^{((\varepsilon))}\left(\left(u^{I}\right)_{I \in G((v)))}:=\left(\varepsilon^{\|I\|} u^{I}\right)_{I \in G((v))}\right.$.

Now we consider the following condition: For some integer $\nu \geq 1$,

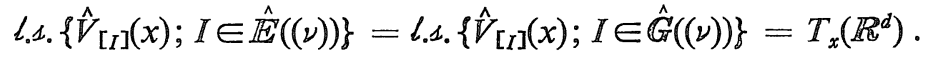

Note that the first equality in (4.7) always holds from (1.5) (ii). We denote by $\nu_{0}=\nu_{0}(x)$ the smallest $\nu$ satisfying (4.7). The following proposition is due to Kusuoka-Stroock ([9]):

Proposition 4.8. If the condition (4.7) is satisfied at $x \in \mathbb{R}^{d}$, then $X^{8}(1, x) \in$ $\mathbb{D}^{\circ}\left(\mathbb{R}^{d}\right)$ is non-degenerate in the Malliavin sense. More precisely, there exist a positive integer $k$ depending only on $\nu_{0}=\nu_{0}(x)$ and, for each $p \geq 1$, a positive constant $c=c(p, x)$ such that

$$
\left\|\left(\operatorname{det} \sigma_{X^{\varepsilon}(1, x)}\right)^{-1}\right\|_{p} \leq c \varepsilon^{-2 k} \quad \text { for all } \varepsilon>0 .
$$

Here $\sigma_{X^{\varepsilon}(1, x)}$ stands for the Malliavin covariance of $X^{\varepsilon}(1, x)$.

Thus, if the condition (4.7) is satisfied at $x \in \mathbb{R}^{d}$, then for any $T \in \mathcal{S}^{\prime}\left(\mathbb{R}^{d}\right)$, $T\left(X^{\varepsilon}(1, x)\right) \in \mathbb{D}^{-\infty}$ is defined for every $\varepsilon>0$ (cf. [4], [16], [17]). In particular, $\delta_{y}\left(X^{\varepsilon}(1, x)\right)$ is defined for every $y \in \mathbb{R}^{d}$ and the generalized expectation $E\left[\delta_{y}\left(X^{\varepsilon}(1, x)\right)\right]$ coincides with $p\left(\varepsilon^{2}, x, y\right)$, where $p(t, x, y)$ is the fundamental solution of the heat equation $\frac{\partial}{\partial t}=\mathbb{L}$.

\section{§5. The Asymptotic Expansion of $\widehat{O}_{x}\left(\mathbb{X}^{2}(\mathbb{1}, x)\right)$ as $£ \downarrow \mathbb{D}$}

We shall continue working in the preceding section. Throughout this section, we fix $x_{0} \in \mathbb{R}^{d}$ and set for simplicity:

$$
\begin{aligned}
& X^{\varepsilon}(t):=X^{\varepsilon}\left(t, x_{0}\right), \quad F_{\nu}^{\varepsilon}(t):=F_{\nu}^{q}\left(t, x_{0}\right), \quad R_{\nu}^{\varepsilon}(t):=R_{\nu}^{\varepsilon}\left(t, x_{0}\right), \\
& \alpha_{\nu}:=\alpha_{\nu}\left(x_{0}\right) \in \mathbb{R}^{d} \otimes \mathbb{R}^{r((\nu))}, \quad M_{\nu}(\cdot):=M_{\nu}\left(x_{0}, \circ\right) \in C^{\infty}\left(\mathbb{R}^{r}((\nu)), \mathbb{R}^{d} \otimes \mathbb{R}^{d}\right), \\
& F_{\nu}(\cdot):=F_{\nu}\left(x_{0},{ }^{\circ}\right)=M_{\nu}(\cdot) \alpha_{\nu} \cdot \in C^{\infty}\left(\mathbb{R}^{r((\nu))}, \mathbb{R}^{d}\right) .
\end{aligned}
$$


Suppose that for some $\nu \geq 1$, the condition (4.7) is satisfied at $x_{0} \in \mathbb{R}^{d}$. In this section, we study the asymptotic expansion of $\delta_{x_{0}}\left(X^{\varepsilon}(1)\right)$ in $\widetilde{\mathbb{D}^{-\infty}}$ as $\varepsilon \downarrow 0$. $\quad \nu_{0}=$ $\nu_{0}\left(x_{0}\right)$ is the smallest integer $\nu$ satisfying (4.7), i.e., it is a natural number such that

$$
\text { l.1. }\left\{\hat{V}_{[I]}\left(x_{0}\right) ; I \in \hat{G}\left(\left(\nu_{0}-1\right)\right)\right\} \subsetneq \text { l.1. }\left\{\hat{V}_{[I]}\left(x_{0}\right) ; I \in \hat{G}\left(\left(\nu_{0}\right)\right)\right\}=T_{x_{0}}\left(\mathbb{R}^{d}\right) .
$$

From this, we can find an $\mathbb{H} \subset \hat{G}\left(\left(\nu_{0}\right)\right)$ with $\frac{\mathrm{w}}{\tilde{T}} \mathbb{H}=d$ such that for each $a=1, \cdots, \nu_{0}$

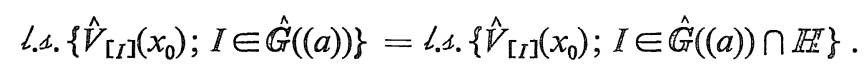

We fix such an $\mathbb{H}$ to proceed with our discussion. Set

$$
\beta:=\left(V_{[I]}^{i}\left(x_{0}\right)\right)_{i \in\{1, \cdots, d\}, I \in H} \in \mathbb{R}^{d} \otimes \mathbb{R}^{d} .
$$

Clearly $\beta$ is invertible. Define $\gamma=\left(\gamma^{I J}\right)_{I \in H, J \in G((\infty))} \in \mathbb{R}^{d} \otimes \mathbb{R}^{\infty}$ as follows:

$$
\gamma:=\beta^{-1}\left(V_{[I]}^{i}\left(x_{0}\right)\right)_{i \in\{1, \cdots, d\}, I \in G((\infty)))} .
$$

Then, from the choice of $\mathbb{H}, r$ has the following properties:

$$
\begin{cases}r^{I J}=\delta_{J}^{I} & \text { if } \quad J \in \mathbb{H}, \\ r^{I J}=0 & \text { if } \quad\|\mathbb{I}\|>\|J\| \text { and } J \in \hat{G}\left(\left(\nu_{0}\right)\right) .\end{cases}
$$

In the following, unless otherwise stated, we assume that $\nu \geq \nu_{0}$. Set

$$
r_{((v))}:=\left(\gamma^{I J}\right)_{I \in H, J \in G((v))}, \quad \hat{r}_{((v))}:=\left(\gamma^{I J}\right)_{I \in H, J \in \hat{G}((v))} .
$$

Then $\hat{\gamma}_{((v))}\left(\hat{\gamma}_{((v))}\right)^{*}>0$ by (5.3). Recalling the definition of $M_{\nu}(\cdot)$ and $F_{\nu}(\cdot)$ given in $\S 4$, we easily see that

$$
M_{\nu}(0)=\mathbb{I}_{\mathbb{R}^{d}}, \quad\left(\partial_{I} \mathbb{F}_{\nu}^{i}(0)\right)_{i \in\{1, \cdots, d\}, I \in \hat{G}\left(\left(\nu_{0}\right)\right)}=\beta \hat{\gamma}_{\left(\left(\nu_{0}\right)\right)} .
$$

Thus, we can choose a small $\kappa_{\nu}>0$ such that for any $u \in \mathbb{R}^{r((\nu))}$ such as $|u| \leq \kappa_{\nu}$

$$
\left\{\begin{array}{l}
\left(\partial_{I} F_{\nu}^{i}(u)\right)_{i \in\{1, \cdots, d\}, I \in \hat{G}_{i}\left(\left(\nu_{0}\right)\right)}\left(\left(\partial_{I} F_{\nu}^{i}(u)_{\left.i \in\{1, \cdots, d\}, I \in \hat{G}\left(\left(\nu_{0}\right)\right)\right)^{*}}\right.\right. \\
\geq \frac{1}{2} \beta \hat{r}_{\left(\left(\nu_{0}\right)\right)}\left(\beta \hat{r}_{\left.\left(\left(\nu_{0}\right)\right)\right)^{*}>0}\right.
\end{array}\right.
$$

$$
\operatorname{det} M_{\nu}(u) \geq \frac{1}{2}
$$

Firstly, from (5.4) we shall present the following lemma: For this, let $\left.\hat{U}_{t}^{((\nu))}:=\left(U_{t}^{I}\right)_{I \in \hat{G}((\nu))} \in \mathbb{D}^{\infty}\left(\mathbb{R}^{r((\nu))-1}\right)\right)$ and set

$$
\hat{\pi}_{((v))}:=\inf \left\{\left\langle\hat{\sigma}_{((v))} \hat{l}, \hat{l}\right\rangle ; \hat{l}=\left(l^{I}\right)_{I \in \hat{G}((v))} \text { such as } \sum_{I \in \hat{G}(\nu))}\left(l^{I}\right)^{2}=1\right\} .
$$




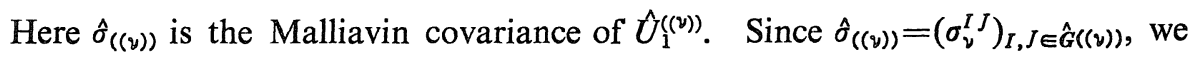
see from (3.8) that $\dot{\pi}_{((v))}>0$ a.s. $(P)$ and

$$
\left(\hat{\pi}_{((\nu))}\right)^{-1} \in L_{\text {oo- }} .
$$

Also, from (3.6) we see

$$
U_{t}^{((\nu))}=\left[\begin{array}{c}
t \\
\hat{U}_{t}^{((v))}
\end{array}\right] .
$$

Now, for simplicity, let us denote by $\sigma_{\nu}^{\varepsilon}$ the Malliavin covariance of $F_{\nu}^{\varepsilon}(1)$. Then

Lemma 5.8. For $0<\varepsilon \leq 1$, it holds that

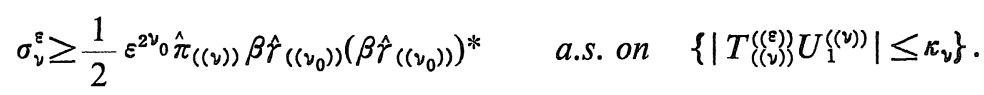

Proof. Let $0<\varepsilon \leq 1$. Since, by (4.6) and (5.7)

$$
\begin{aligned}
D\left(F_{\nu}^{\mathrm{\varepsilon}, i}(1)\right) & =\sum_{I \in G((\nu))} \partial_{I} F_{\nu}^{i}\left(T_{((\nu))}^{((\varepsilon))} U_{1}^{((\nu))}\right) \varepsilon^{\|I\|} D U_{1}^{I} \\
& =\sum_{I \in \hat{G}((\nu))} \partial_{I} F_{\nu}^{i}\left(T_{((\nu))}^{(\mathcal{\varepsilon}))} U_{1}^{((\nu))}\right) \varepsilon^{\|I\|} D U_{1}^{I} \quad i \in\{1, \cdots, d\},
\end{aligned}
$$

we observe that for $i, j \in\{1, \cdots, d\}$

$$
\begin{aligned}
& \left(\sigma_{\nu}^{\varepsilon}\right)^{i j}=\left\langle D\left(F_{\nu}^{\varepsilon, i}(1)\right), D\left(F_{\nu}^{\varepsilon, j}(1)\right)\right\rangle \\
& =\sum_{I, J \in \hat{G}((\nu))} \partial_{I} F_{\nu}^{i}\left(T_{((\nu))}^{((\varepsilon))} U_{1}^{((\nu))}\right) \partial_{J} F_{\nu}^{j}\left(T_{((\nu))}^{((\varepsilon))} U_{1}^{((\nu))}\right) \varepsilon^{\|I\|+\|J\|} \sigma_{\nu}^{I J} .
\end{aligned}
$$

Hence, for any $l \in \mathbb{R}^{d}$ it holds that

$$
\begin{aligned}
& \left\langle\sigma_{\nu}^{\varepsilon} l, l\right\rangle=\sum_{i, j=1}^{d}\left(\sigma_{\nu}^{\varepsilon}\right)^{i j} l^{i} l^{j}
\end{aligned}
$$

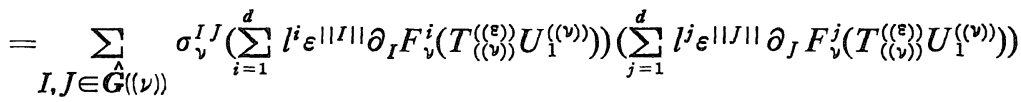

$$
\begin{aligned}
& \geq \hat{\pi}_{((\nu))} \sum_{I \in \hat{G}((\nu))} \varepsilon^{2|| I \|}\left(\sum_{i=1}^{d} l^{i} \partial_{I} F_{\nu}^{i}\left(T_{((\nu))}^{(\varepsilon))} U_{1}^{((\nu))}\right)\right)^{2} \\
& \geq \hat{\pi}_{((\nu))} \varepsilon^{2 \nu_{0}} \sum_{I \in \hat{G}\left(\left(\nu_{0}\right)\right)}\left(\sum_{i=1}^{d} l^{i} \partial_{I} F_{\nu}^{i}\left(T_{((\nu))}^{(\varepsilon))} U_{1}^{((\nu))}\right)\right)^{2} \\
& =\varepsilon^{2 \nu_{0}} \hat{\pi}_{((\nu))} \mid\left(\left(\partial_{I} F_{\nu}^{i}\left(T_{((\nu))}^{((\varepsilon))} U_{1}^{((\nu))}\right)_{\left.i \in\{1, \cdots, d\}, I \in \hat{G}_{\left(\left(\nu_{0}\right)\right)}\right)\left.* l\right|^{2} .} .\right.\right.
\end{aligned}
$$

Consequently, combining this with (5.4), we obtain (5.8). //

The following is an immediate consequence of (5.6) and (5.8): For $0<\varepsilon \leq 1$, det $\sigma_{\vartheta}^{\varepsilon}>0$ a.s. on $\left\{\left|T_{((\nu))}^{((\varepsilon))} U_{1}^{((\nu))}\right| \leq \kappa_{y}\right\}$ and 


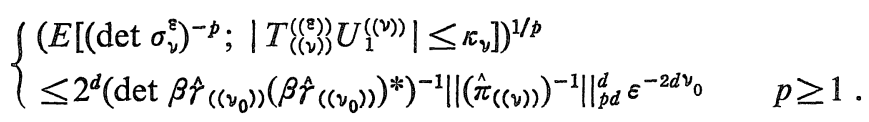

Secondly, we choose an $h \in C^{\circ \infty}\left(\mathbb{R}^{1}\right)$ such that $0 \leq h \leq 1, h(x)=1$ if $|x| \leq \frac{1}{2}$ and $h(x)=0$ if $|x| \geq 1$. Set $h_{\nu}(x):=h\left(x /\left(\kappa_{\nu} / 2\right)^{2}\right)$ and define $\chi_{\nu}^{\varepsilon} \in \mathbb{D}^{\infty}$ by

$$
\chi_{\nu}^{\varepsilon}:=h_{\nu}\left(\left|T_{((\nu))}^{((\mathrm{q}))} U_{1}^{((\nu))}\right|^{2}\right) .
$$

Let $\delta_{0}$ be the Dirac delta-function at $0 \in \mathbb{R}^{d}$. Then $\delta_{0}\left(X^{\mathrm{g}}(1)-x_{0}\right), \chi_{\nu}^{\mathrm{q}} \cdot \delta_{0}\left(X^{\mathfrak{q}}(1)\right.$ $\left.-x_{0}\right) \in \widetilde{\mathbb{D}^{-\infty}}$ (in fact, $\in \bigcap_{p>1} \mathbb{D}_{p}^{-2([d / 2]+1)}$ ). By (4.8) and (3.14), we can prove the following in the same way as in [17]:

Lemma 5.10. For any $\nu \geq \nu_{0}$ and $p>1$, there exist positive constants $c_{1}$ and $c_{2}$ independent of $\varepsilon$ such that

$$
\left\|\delta_{0}\left(X^{\varepsilon}(1)-x_{0}\right)-\chi_{\nu}^{\varepsilon} \delta_{0}\left(X^{\mathrm{z}}(1)-x_{0}\right)\right\|_{p_{0}-2([d / 2]+1)} \leq c_{1} \exp \left(-\frac{c_{2}}{\varepsilon^{2}}\right) \quad \text { as } \quad \varepsilon \downarrow 0 .
$$

By virtue of (5.9), for any $T \in \mathcal{S}^{\prime}\left(\mathbb{R}^{d}\right), \chi_{\nu}^{\varepsilon} \cdot T\left(F_{\nu}^{\varepsilon}(1)\right) \in \widetilde{\mathbb{D}^{-\infty}}$ is defined similarly to $T\left(X^{\mathbb{q}}(1)-x_{0}\right)$. More precisely, the mapping $\phi \in \mathcal{S}\left(\mathbb{R}^{d}\right) \mapsto \chi_{\nu}^{\mathbb{\varepsilon}} \circ \phi\left(F_{\nu}^{\mathbb{\varepsilon}}(1)\right) \in$ $\mathbb{D}^{\infty}$ can be extended uniquely to a linear mapping

$$
T \in \mathcal{S}^{\prime}\left(\mathbb{R}^{d}\right) \mapsto \chi_{\nu}^{\varepsilon} \cdot T\left(F_{\nu}^{\varepsilon}(1)\right) \in \mathbb{D}^{-\infty}
$$

such that its restriction $T \in \mathcal{I}_{-2 m} \mapsto \chi_{\nu}^{\varepsilon} \cdot T\left(F_{\nu}^{\mathbb{\varepsilon}}(1)\right) \in \mathbb{D}_{p}^{-2 m}$ is continuous for every $p \in(1, \infty)$ and $m=0,1,2, \cdots$ (cf. [4], [16]). In fact, $\chi_{\nu}^{\varepsilon} \cdot T\left(F_{\nu}^{\varepsilon}(1)\right) \in \widetilde{\mathbb{D}^{-\infty}}$ for every $T \in \mathcal{S}^{\prime}\left(\mathbb{R}^{d}\right)$. In particular, if we take $T=\delta_{0}, \chi_{\nu}^{\varepsilon} \circ \delta_{0}\left(F_{\nu}^{\varepsilon}(1)\right) \in \bigcap_{p>1} \mathbb{D}_{p}^{-2([d / 2]+1)}$. From (4.3) and (4.4), we note that

$$
X^{\mathrm{e}}(1)-x_{0}-F_{\nu}^{\mathrm{e}}(1)=O\left(\varepsilon^{\nu+1}\right) \quad \text { in } \mathbb{D}^{\infty}\left(\mathbb{R}^{d}\right) \quad \text { as } \varepsilon \downarrow 0
$$

(cf. [17]). By this, (5.9) and (4.8), we can also prove the following in the same way as in [17]:

Lemma 5.11. There exists an increasing sequence $\left\{l_{\nu}=l_{\nu}\left(d, \nu_{0}\right)\right\}_{\nu \geq v_{0}}$ such that

(i) $\lim _{\nu \uparrow^{\infty}} l_{\nu}=+\infty$,

(ii) for any $p>1$, $\left\|\chi_{\nu}^{\varepsilon} \circ \delta_{0}\left(X^{q}(1)-x_{0}\right)-\chi_{\nu}^{\varepsilon}{ }^{\circ} \delta_{0}\left(F_{\nu}^{\varepsilon}(1)\right)\right\|_{p,-2([d / 2]+2)}=O\left(\varepsilon^{l y}\right)$ as $\varepsilon \downarrow 0$.

Thus, from (5.10) and (5.11), it follows that for any $p>1$ and $\nu \geq \nu_{0}$

$$
\left\|\delta_{0}\left(X^{2}(1)-x_{0}\right)-x_{\nu}^{\imath} \cdot \delta_{0}\left(F_{\nu}^{q}(1)\right)\right\|_{p,-2\left(\left[L_{2} / 2\right]+2\right)}=O\left(\varepsilon^{l} \nu\right) \quad \text { as } \quad \varepsilon \downarrow 0 .
$$


Thirdly, we shall present an available expression of $\chi_{\nu}^{\varepsilon} \cdot \delta_{0}\left(F_{\nu}^{\varepsilon}(1)\right)$. As before, it is assumed that $\nu \geq \nu_{0}$. First of all, we note that for any $G \in \mathbb{D}^{\infty}$,

$$
E\left[G \chi_{\nu}^{\varepsilon} \circ \delta_{0}\left(F_{\nu}^{\varepsilon}(1)\right)\right]=\lim _{\psi \rightarrow \delta_{0}} E\left[G \chi_{\nu}^{\varepsilon} \cdot \psi\left(F_{\nu}^{\varepsilon}(1)\right)\right]
$$

For $\varepsilon>0$, the following matrices are defined:

$$
\begin{aligned}
& A_{((\nu))}^{\varepsilon}:=\left(\varepsilon^{\|J\|-\|I\| \gamma^{I J}}\right)_{I \in H, J \in G((\nu))}, \\
& \hat{A}_{((v))}^{\varepsilon}:=\left(\varepsilon^{\|J\|-\|I\|} \gamma^{I J}\right)_{I \in H_{0} J \in \hat{G}((\nu))}, \\
& a^{\varepsilon}:=\left(A_{((\nu))}^{\varepsilon, I(0)}\right)_{I \in \boldsymbol{H}}=\left(\left.\varepsilon^{2-\|I\|}\right|^{I(0)}\right)_{I \in \boldsymbol{H}}, \\
& \hat{A}_{((v))}:=\left(\delta \|_{|J \||}^{I \|} \gamma^{I J}\right)_{I \in H, J \in \hat{G}((v))}, \\
& B_{((v))}:=\left(\delta_{J}^{I}\right)_{I \in G((v)) \backslash H_{,} J \in G((v))}, \\
& \hat{B}_{((\nu))}:=\left(\delta_{J}^{I}\right)_{I \in \hat{G}((\nu)) \backslash H_{0} J \in \hat{G}((\nu)),}, \\
& T_{\mathbb{H}}^{((\varepsilon))}:=\left(\varepsilon^{\|J\|} \delta_{J}^{I}\right)_{I \in H, J \in H} .
\end{aligned}
$$

Set

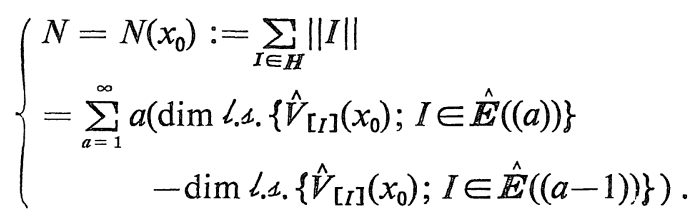

Then, by recalling (5.3), the following is easily verified:

Lemma 5.15. The following holds:

(i) $\alpha_{\nu}=\beta \gamma_{((v))}$.

(ii) $A_{((v))}^{\varepsilon}=\left[\begin{array}{ll}a^{\varepsilon} & \hat{A}_{((v))}^{\varepsilon}\end{array}\right], B_{((v))}=\left[\begin{array}{ll}1 & 0 \\ 0 & \hat{B}_{((v))}\end{array}\right]$ and $\gamma_{((v))} T_{((v))}^{((\varepsilon))}=T_{\mathbb{H}}^{(\varepsilon))} A_{((v)) \text {. }}^{\varepsilon}$

(iii) $\lim _{\varepsilon \downarrow 0} \hat{A}_{((v))}^{\varepsilon}=\hat{A}_{((v))}$.

(iv) $\hat{A}_{((v))}\left(\hat{A}_{((v))}\right)^{*}>0$ and $\left[\begin{array}{l}\hat{A}_{((v))} \\ \hat{B}_{((v))}\end{array}\right]$ is invertible.

(v) $T_{\mathbb{H}}^{((\varepsilon))}$ is invertible and $\operatorname{det} T_{\mathbb{H}}^{((\varepsilon))}=\varepsilon^{N}$.

Thus, from the above (iii) and (iv), there exists an $\varepsilon_{0}=\varepsilon_{0}(\nu)>0$ such that for every $0<\varepsilon \leq \varepsilon_{0}$

$$
\left\{\begin{array}{l}
\hat{A}_{((v))}^{\varepsilon}\left(\hat{A}_{((v))}^{\varepsilon}\right)^{*} \geq \frac{1}{2} \hat{A}_{((v))}\left(\hat{A}_{((v)))}\right)^{*}>0, \\
{\left[\begin{array}{l}
\hat{A}_{((v))}^{\varepsilon} \\
\hat{B}_{((v))}
\end{array}\right]\left[\begin{array}{l}
\hat{A}_{((v))}^{\varepsilon} \\
\hat{B}_{((v))}
\end{array}\right]^{*} \geq \frac{1}{2}\left[\begin{array}{l}
\hat{A}_{((v))} \\
\hat{B}_{((v))}
\end{array}\right]\left[\begin{array}{l}
\hat{A}_{((v))} \\
\hat{B}_{((v))}
\end{array}\right]^{*}>0 .}
\end{array}\right.
$$

And, from (ii), it is easy to see that 


$$
\left\{\begin{array}{l}
{\left[\begin{array}{l}
A_{((v))}^{\varepsilon} \\
B_{((v))}
\end{array}\right] U_{1}^{((v))}=\left[\begin{array}{c}
A_{((v))}^{\varepsilon} U_{1}^{((v))} \\
1 \\
\hat{B}_{((v))} \hat{U}_{1}^{(v))}
\end{array}\right], \quad A_{((v))}^{\varepsilon} U_{1}^{((v))}=a^{\varepsilon}+\hat{A}_{((v))}^{\varepsilon} \hat{U}_{1}^{((v))},} \\
{\left[\begin{array}{l}
A_{((v))}^{\varepsilon} U_{1}^{((v))} \\
\hat{B}_{((v))} \hat{U}_{1}^{((v))}
\end{array}\right]=\left[\begin{array}{c}
a^{\varepsilon} \\
0
\end{array}\right]+\left[\begin{array}{l}
\hat{A}_{((v))}^{\varepsilon} \\
\hat{B}_{((v))}
\end{array}\right] \hat{U}_{1}^{((v))} .}
\end{array}\right.
$$

Consequently, putting (5.6), (5.16) and (5.17) together, we have that

$$
A_{((v))}^{\varepsilon} U_{1}^{((v))} \text { and }\left[\begin{array}{ll}
A_{((\nu))}^{\varepsilon} & U_{1}^{((v))} \\
\hat{B}_{((v))} & \hat{U}_{1}^{((v))}
\end{array}\right] \text { are uniformly non-degenerate }
$$

(cf. [17]). Also, from (4.6), (5.15) (i), (ii), (5.16) and (5.17), it is easily seen that

$$
F_{\nu}^{\varepsilon}(1)=M_{\nu}\left(T_{((\nu))}^{(\varepsilon))}\left(C_{((v))}^{\varepsilon}\right)^{-1}\left[\begin{array}{c}
A_{((v))}^{\varepsilon} U_{1}^{((v))} \\
1 \\
\hat{B}_{((v))} \hat{U}_{1}^{((v))}
\end{array}\right]\right) \beta T_{\mathbb{H I}}^{((\varepsilon))} A_{((v))}^{\varepsilon} U_{1}^{((v))} \quad 0<\varepsilon \leq \varepsilon_{0}
$$

where $C_{((v))}^{\varepsilon}:=\left[\begin{array}{l}A_{((v))}^{\varepsilon} \\ B_{((v))}\end{array}\right]$ is invertible for $0<\varepsilon \leq \varepsilon_{0}$ by $(5.16)$.

Now, as to $\chi_{\nu}^{\varepsilon} \delta_{0}\left(F_{\nu}^{\varepsilon}(1)\right)$, we present the following: Set $f_{\nu} \in C_{0}^{\infty}\left(\mathbb{R}^{r((\nu))}\right)$ as follows:

$$
f_{\nu}(u):=\frac{h_{\nu}\left(|u|^{2}\right)}{\operatorname{det} M_{\nu}(u)} \quad u \in \mathbb{R}^{r(v))}
$$

which is well-defined from (5.5) and the definition of $h_{v}$. Then we have

Lemma 5.20. For each $0<\varepsilon \leq \varepsilon_{0}$

$$
\chi_{\nu}^{\varepsilon} \cdot \delta_{0}\left(F_{\nu}^{\varepsilon}(1)\right)=\varepsilon^{-N}|\operatorname{det} \beta|^{-1} f_{\nu}\left(T_{((\nu))}^{(\varepsilon))} U_{1}^{((\nu))}\right) \circ \delta_{0}\left(A_{((\nu))}^{\varepsilon} U_{1}^{(\nu))}\right),
$$

where $\delta_{0}$ is the Dirac delta-function at $0 \in \mathbb{R}^{d}=\mathbb{R}^{*} \mathbb{H}$.

Proof. Let $0<\varepsilon \leq \varepsilon_{0}$. For simplicity, set

$$
\begin{aligned}
T & :=T_{((v))}^{((\varepsilon))}, \quad T_{\mathbb{H}}:=T_{\mathbb{H}}^{((\varepsilon))}, \quad C:=C_{((v))}^{\varepsilon}, \\
V & :=A_{((v))}^{\varepsilon} U_{1}^{((v))} \in\left\{\left(v^{I}\right)_{I \in \mathbb{H}}\right\} \simeq \mathbb{R}^{d}, \\
W & :=\hat{B}_{((v))} \hat{U}_{1}^{((v))} \in\left\{\left(w^{I}\right)_{I \in \hat{G}((v)) \backslash \mathbb{H}\}} \simeq \mathbb{R}^{r((v))-1-d} .\right.
\end{aligned}
$$

Choose a $\psi \in C_{0}^{\infty}\left(\mathbb{R}^{d}\right)$ so that $\psi \geq 0$ and $\int_{\mathbb{R}^{d}} \psi(v) d v=1$. For $\lambda>0$, set $\psi_{\lambda}(v)$ $:=1 / \lambda^{d} \psi(v / \lambda)$. Let $G \in \mathbb{D}^{\infty}$ be fixed arbitrarily. By (5.13),

$$
E\left[G \chi_{\nu}^{\varepsilon} \circ \delta_{0}\left(F_{\nu}^{3}(1)\right)\right]=\lim _{\lambda \downarrow 0} E\left[G \chi_{\nu}^{3} \cdot \psi_{\lambda}\left(F_{\nu}^{\varepsilon}(1)\right)\right] .
$$

On the other hand, by (5.19) we observe that 


$$
\begin{aligned}
& E\left[G \chi_{\nu}^{\varepsilon} \cdot \psi_{\lambda}\left(F_{\nu}^{\varepsilon}(1)\right)\right] \\
& =E\left[G h_{y}\left(\left|T C^{-1}\left[\begin{array}{c}
V \\
1 \\
W
\end{array}\right]\right|^{2}\right) \psi_{\lambda}\left(M_{y}\left(T C^{-1}\left[\begin{array}{c}
V \\
1 \\
W
\end{array}\right]\right) \beta T_{H} V\right)\right] \\
& =\int_{\boldsymbol{R}^{d}} \int_{\boldsymbol{R}^{r((\nu))-1-d}} h_{\nu}\left(\left|T C^{-1}\left[\begin{array}{c}
v \\
1 \\
w
\end{array}\right]\right|^{2}\right) \psi_{\lambda}\left(M_{\nu}\left(T C^{-1}\left[\begin{array}{c}
v \\
1 \\
w
\end{array}\right]\right) \beta T_{\boldsymbol{H} v)}\right. \\
& \times\left\langle G, \delta_{\left[\begin{array}{l}
v \\
{ }_{w}
\end{array}\right]}\left(\begin{array}{l}
V \\
W
\end{array}\right)\right\rangle d v d w \\
& =\varepsilon^{-N} \int_{\boldsymbol{R}^{d}} \int_{\boldsymbol{R}^{r((v))-1-d}} h_{\nu}\left(\left|T C^{-1}\left[\begin{array}{c}
\left(T_{\boldsymbol{H}}\right)^{-1} v \\
1 \\
w
\end{array}\right]\right|^{2}\right) \\
& \times \psi_{\lambda}\left(M_{v}\left(T C^{-1}\left[\begin{array}{c}
\left(T_{\boldsymbol{H}}\right)^{-1} v \\
1 \\
w
\end{array}\right]\right) \beta v\right)\left\langle G, \delta\left[\begin{array}{c}
\left(T_{\boldsymbol{H}}\right)^{-1} v \\
w
\end{array}\right]\left(\begin{array}{c}
V \\
W
\end{array}\right)\right\rangle d v d w
\end{aligned}
$$

(by a change of variables: $v \mapsto\left(T_{\boldsymbol{H}}\right)^{-1} v$ and (5.15) (v))

$$
\begin{aligned}
=\varepsilon^{-N} \int_{\boldsymbol{R}^{d}} & \int_{\boldsymbol{R}^{r((v))-1-d}} h_{y}\left(\left|T C^{-1}\left[\begin{array}{c}
\left(T_{\boldsymbol{H}}\right)^{-1} v \\
1 \\
w
\end{array}\right]\right|^{2}\right) \\
& \times \frac{1}{\lambda^{d}} \psi\left(M_{v}\left(T C^{-1}\left[\begin{array}{c}
\left(T_{\boldsymbol{H}}\right)^{-1} v \\
1 \\
w
\end{array}\right]\right) \beta \frac{v}{\lambda}\right)\left\langle G, \delta\left[\begin{array}{c}
\left(T_{\boldsymbol{H}}\right)^{-1} v \\
w
\end{array}\right]\left(\begin{array}{l}
V \\
W
\end{array}\right)\right\rangle d v d w
\end{aligned}
$$

(by the definition of $\psi_{\lambda}$ )

$$
\begin{aligned}
=\varepsilon^{-N} \int_{\boldsymbol{R}^{d}} \int_{\boldsymbol{R}^{r((v))-1-d}} h_{\nu}\left(\left|T C^{-1}\left[\begin{array}{c}
\left(T_{\boldsymbol{H}}\right)^{-1} \lambda v \\
1 \\
w
\end{array}\right]\right|^{2}\right) \\
\quad \times \psi\left(M_{\nu}\left(T C^{-1}\left[\begin{array}{c}
\left(T_{\boldsymbol{H}}\right)^{-1} \lambda v \\
1 \\
w
\end{array}\right]\right) \beta v\right)\left\langle G, \delta_{\left[\begin{array}{c}
\left(T_{H}\right)^{-1} \lambda v \\
w
\end{array}\right]}\left(\begin{array}{l}
V \\
W
\end{array}\right)\right\rangle d v d w
\end{aligned}
$$

(by a change of variables: $v \mapsto \lambda v$ ).

Hence, letting $\lambda \downarrow 0$, we see

$$
\begin{aligned}
& E\left[G \chi_{\nu}^{\varepsilon} \cdot \delta_{0}\left(F_{\nu}^{\varepsilon}(1)\right)\right] \\
& =\varepsilon^{-N} \int_{R^{d}} \int_{R^{r}(v)-1-d} h_{\nu}\left(\left|T C^{-1}\left[\begin{array}{c}
0 \\
1 \\
w
\end{array}\right]\right|^{2}\right) \\
& \quad \times \psi\left(M_{\nu}\left(T C^{-1}\left[\begin{array}{c}
0 \\
1 \\
w
\end{array}\right]\right) \beta v\right)\left\langle G, \delta_{\left[\begin{array}{l}
0 \\
w
\end{array}\right]}\left(\begin{array}{l}
V \\
W
\end{array}\right)\right\rangle d v d w \\
& =\varepsilon^{-N} \int_{R^{r((v))-1-d}} h_{\nu}\left(\left|T C^{-1}\left[\begin{array}{c}
0 \\
1 \\
w
\end{array}\right]\right|^{2}\right)\left\langle G, \delta_{\left.\left[\begin{array}{l}
0 \\
w
\end{array}\right]\left(\begin{array}{l}
V \\
W
\end{array}\right)\right\rangle d w}\right.
\end{aligned}
$$




$$
\begin{gathered}
\times \int_{\mathbb{R}^{d}} \psi\left(M_{y}\left(T C^{-1}\left[\begin{array}{c}
0 \\
1 \\
w
\end{array}\right]\right) \beta v\right) d v \\
=\varepsilon^{-N} \int_{\mathbb{R}^{r(v))-1-d}} h_{v}\left(\left|T C^{-1}\left[\begin{array}{c}
0 \\
1 \\
w
\end{array}\right]\right|^{2}\right)\left\langle G, \delta_{\left[{ }^{0}\right]}\left(\begin{array}{l}
V \\
W
\end{array}\right)\right\rangle \\
\times|\operatorname{det} \beta|^{-1}\left(\operatorname{det} M_{v}\left(T C^{-1}\left[\begin{array}{c}
0 \\
1 \\
w
\end{array}\right]\right)\right)^{-1} d w
\end{gathered}
$$

(by a change of variables: $v \mapsto\left(M_{\nu}\left(T C^{-1}\left[\begin{array}{l}0 \\ 1 \\ w\end{array}\right]\right) \beta\right)^{-1} v$ and by the fact:

$\left.\int_{\mathbb{R}^{d}} \psi(v) d v=1\right)$

$$
=\varepsilon^{-N}|\operatorname{det} \beta|^{-1} \int_{R^{r((v))-1-d}} f_{\nu}\left(T C^{-1}\left[\begin{array}{c}
0 \\
1 \\
w
\end{array}\right]\right)\left\langle G, \delta_{\left[{ }_{w}^{0}\right]}\left(\begin{array}{l}
V \\
W
\end{array}\right)\right\rangle d w
$$

(by the definition of $f_{y}$ )

$$
=\varepsilon^{-N}|\operatorname{det} \beta|^{-1}\left\langle G, f_{\nu}\left(T U_{1}^{(\nu))}\right) \cdot \int_{\mathbb{R}^{r((v))-1-d}} \delta_{\left[\begin{array}{l}
0 \\
w
\end{array}\right]}\left(\begin{array}{l}
V \\
W
\end{array}\right) d w\right\rangle .
$$

Here the last equality has come from the fact:

$$
\begin{aligned}
& f_{\nu}\left(T C^{-1}\left[\begin{array}{c}
0 \\
1 \\
w
\end{array}\right]\right)\left\langle G, \delta_{\left[\begin{array}{l}
0 \\
w
\end{array}\right]}\left(\begin{array}{l}
V \\
W
\end{array}\right)\right\rangle=\left\langle G, f_{v}\left(T C^{-1}\left[\begin{array}{c}
V \\
1 \\
W
\end{array}\right]\right) \delta_{\left[\begin{array}{l}
0 \\
w
\end{array}\right]}\left(\begin{array}{l}
V \\
W
\end{array}\right)\right\rangle \\
& =\left\langle G, f_{\nu}\left(T U_{1}^{(\nu))} \delta_{\left[\begin{array}{l}
0 \\
w
\end{array}\right]}\left(\begin{array}{l}
V \\
W
\end{array}\right)\right\rangle .\right.
\end{aligned}
$$

Thus, since $G \in \mathbb{D}^{\infty}$ is arbitrary, we obtain

$$
\chi_{\nu}^{\varepsilon} \cdot \delta_{0}\left(F_{\nu}^{\mathcal{q}}(1)\right)=\varepsilon^{-N}|\operatorname{det} \beta|^{-1} f_{\nu}\left(T U_{1}^{((\nu))}\right) \cdot \int_{\mathbb{R}^{r((\nu))-1-d}} \delta_{\left[\begin{array}{l}
0 \\
w
\end{array}\right]}\left(\begin{array}{l}
V \\
W
\end{array}\right) d w,
$$

from which and the fact:

$$
\int_{\boldsymbol{R}^{r((v))-1-d}} \delta_{\left[\begin{array}{l}
0 \\
w
\end{array}\right]}\left(\begin{array}{l}
V \\
W
\end{array}\right) d w=\delta_{0}(V),
$$

(5.20) follows at once. //

Noting that $\hat{V}_{0}\left(x_{0}\right)=\sum_{I \in \boldsymbol{H}} \gamma^{I(0)} \hat{V}_{[I]}\left(x_{0}\right)$, we define

$$
\mu_{0}:=\max \left\{\|I\| ; \gamma^{I(0)} \neq 0\right\}
$$

Here $\max \{\phi\}:=0$ for convenience. From its definition, the following is clear: 


$$
\left\{\begin{array}{l}
\text { (i) } \gamma^{I(0)}=0 \text { for } I \in \mathbb{H} \text { such as }\|I\|>\mu_{0} . \\
\text { (ii) If } \mu_{0} \geq 1 \text {, then } \gamma^{I(0)} \neq 0 \text { for some } I \in \mathbb{H} \text { with }\|I\|=\mu_{0} .
\end{array}\right.
$$

In the following, individually, we think of the case (A) and (B):

$$
\left\{\begin{array}{l}
\text { (A) The case where } \mu_{0} \leq 2 . \\
\text { (B) The case where } \mu_{0} \geq 3 .
\end{array}\right.
$$

First, we consider the case (A). From (5.20) we shall present the asymptotic expansion of $\chi_{\nu}^{\varepsilon} \cdot \delta_{0}\left(F_{\nu}^{\varepsilon}(1)\right)$ in $\widetilde{\mathbb{D}^{-\infty}}$ as $\varepsilon \downarrow 0$ (cf. [17]): Note that by (5.22) (i), $\lim _{\varepsilon, 0} a^{\varepsilon}=a:=\left(\delta_{2}^{\|I\|} r^{I(0)}\right)_{I \in H}$. We define $v_{a}=\left(v_{a}^{I}\right)_{I \in H}, v_{a}^{((v))}=\left(v_{a}^{((v)) I}\right)_{I \in H} \in \mathbb{D}^{\infty}\left(\mathbb{R}^{d}\right)$ and $\Xi_{a} \in \widetilde{\mathbb{D}^{-\infty}}, a \geq 0$ as follows:

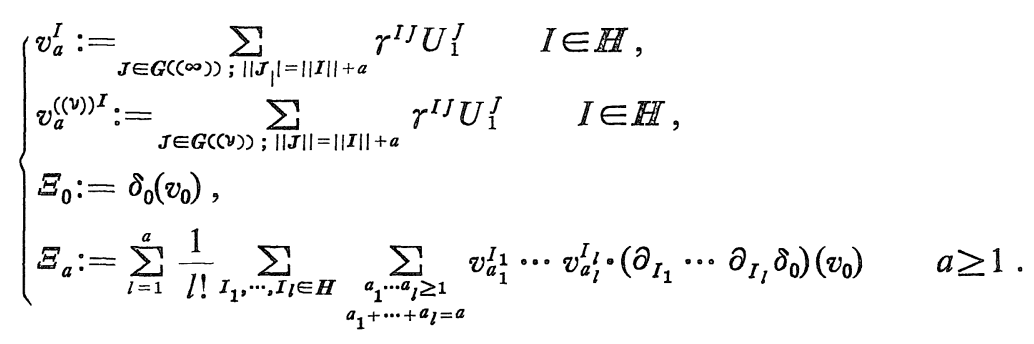

Putting $v_{a}^{((v))}$ in place of $v_{a}$, we similarly define $\Xi_{a}^{((\nu))} \in \widetilde{D^{-\infty}}, a \geq 0$. Since

$$
v_{0}=v_{0}^{((v))}=a+\hat{A}_{\left(\left(v_{0}\right)\right)} \hat{U}_{1}^{\left(\left(v_{0}\right)\right)},
$$

$v_{0}=v_{0}^{((v))} \in \mathbb{D}^{\infty}\left(\mathbb{R}^{d}\right)$ is non-degenerate by (5.6) and (5.15) (iv), and so, $\Xi_{a}, \Xi_{a}^{((v))}$, $a \geq 0$ are well-defined. Note that $v_{a}^{((\nu))}=v_{a}$ for $0 \leq a \leq \nu-\nu_{0}$ and hence

$$
\Xi_{a}^{((\nu))}=\Xi_{a} \quad \text { for any } 0 \leq a \leq \nu-\nu_{0} .
$$

From (5.3), (5.22) (i) and the definition of $A_{((v))}^{\varepsilon}$, it is easy to see that

$$
A_{((v))}^{\varepsilon} U_{1}^{((\nu))}=\sum_{a=0}^{\nu-1} \varepsilon^{a} v_{a}^{((\nu))} .
$$

Thus, by applying the general theory due to S. Watanabe ([17]), from this and (5.18), it follows that

$$
\delta_{0}\left(A_{(\nu))}^{\varepsilon} U_{1}^{((\nu))}\right) \sim \Xi_{0}^{((v))}+\varepsilon \Xi_{1}^{((\nu))}+\varepsilon^{2} \Xi_{2}^{((v))}+\cdots \quad \text { in } \widetilde{\mathbb{D}^{-\infty}} \text { as } \varepsilon \downarrow 0 .
$$

Next, as to the asymptotic expansion of $f_{v}\left(T_{\left(\begin{array}{l}(\xi)) \\ (y)\end{array}\right)}^{((\nu))}\right)$, we easily see that it is given by

$$
f_{v}\left(T_{((v))}^{((\ell))} U_{1}^{((v))}\right) \sim \xi_{0}^{((v))}+\varepsilon \xi_{1}^{((v))}+\varepsilon^{2} \xi_{2}^{((v))}+\ldots \quad \text { in } \mathbb{D}^{\infty} \text { as } \varepsilon \downarrow 0,
$$

where $\xi_{a}^{((v))} \in \mathbb{D}^{\infty}, a \geq 0$ are defined as follows: 


$$
\begin{aligned}
& \xi_{0}^{((v))}:=1, \\
& \xi_{a}^{((\nu))}:=\sum_{l=1}^{e} \frac{1}{l !} \sum_{\substack{I_{1}, \cdots, I_{l} \in G((\nu)) \\
\left\|I_{1}\right\|+\cdots+\left\|I_{l}\right\|=a}}\left(\partial_{I_{1}} \ldots \partial_{I_{l}} f_{v}\right)(0) U_{1}^{I} \ldots U_{1}^{I} l \\
& =\sum_{l=1}^{a} \frac{1}{l !} \sum_{\substack{\left.I_{1}, \cdots, I_{l} \in G(\mathcal{G})\right) \\
\left\|I_{1}\right\|+\ldots+\left\|I_{l}\right\|=a}}\left(\partial_{I_{1}} \ldots \partial_{I_{l}} \frac{1}{\operatorname{det} M_{\nu}}\right)(0) U_{1}^{I_{1}} \ldots U_{1^{l}}^{I_{l}} \quad a \geq 1 .
\end{aligned}
$$

Therefore, putting (5.20), (5.25) and (5.26) together, we have the asymptotic expansion of $\chi_{\nu}^{\varepsilon} \cdot \delta_{0}\left(F_{\nu}^{\varepsilon}(1)\right)$ :

$$
\begin{aligned}
& \chi_{\nu}^{\varepsilon} \circ \delta_{0}\left(F_{\nu}^{\varepsilon}(1)\right) \sim \varepsilon^{-N}|\operatorname{det} \beta|^{-1}\left(\Xi_{0}^{((\nu))}+\sum_{a=1}^{\infty} \varepsilon^{a} \sum_{\substack{b, c \geqq 0 \\
b+c=a}} \xi_{b}^{((\nu))} \circ \Xi_{c}^{((\nu))}\right) \\
& \text { in } \widetilde{\mathbb{D}^{-\infty}} \text { as } \varepsilon \downarrow 0 \text {. }
\end{aligned}
$$

Now, as to $M_{\nu}$, we make a few remarks: Here, for a moment let $\nu \geq 1$. Define $e_{J_{1}, \cdots, J_{d}}(I)$ for $I \in \mathbb{E}(\infty), J_{1}, \cdots, J_{d} \in\{\phi\} \cup \mathbb{E}(\infty)$ by

$$
e_{J_{1}, \cdots, J_{d}}(I):= \begin{cases}1 & \text { if } J_{i}=\mathbb{I} \text { and } J_{1}, \cdots, J_{i-1}, J_{i+1}, \cdots, J_{d}=\phi \\ & \text { for some } i \in\{1, \cdots, d\} \\ 0 & \text { otherwise. }\end{cases}
$$

Then it is easy to see that for any $I_{1}, \cdots, \mathbb{I}_{a} \in \mathbb{E}(\infty)$ and $a \geq 1$

$$
\left\{\begin{array}{l}
\partial_{I_{1}} \cdots \partial_{I_{a}} \operatorname{det} M_{\nu} \\
=\sum_{J_{1}^{(1)}, \ldots, J_{d}^{(1)}, \ldots, J_{1}^{(a)}, \ldots, J_{d}^{(a)} \in\{\phi\} \cup E^{(\infty)}} e_{J_{1}^{(1)}, \ldots, J_{d}^{(1)}}\left(I_{1}\right) \cdots e_{J_{1}^{(a)}, \ldots, J_{d}^{(a)}\left(I_{a}\right)} \\
\quad \times \operatorname{det}\left[\partial_{J_{1}^{(1)}} \cdots \partial_{J_{1}^{(a)}} M_{\nu, 1}, \cdots, \partial_{J_{d}^{(1)}} \cdots \partial_{J_{d}^{(a)}} M_{\left.\nu_{,}\right]}\right] .
\end{array}\right.
$$

Here $M_{v, j}$ denotes the $j$-th column of $M_{v}$ and we set $\partial_{\phi}:=$ the identity operator if $J=\phi$. On the other hand, from the definition of $M_{v}$, we also easily see that for $0 \leq a \leq \nu-1, J_{1}, \cdots, J_{a} \in\{\phi\} \cup \mathbb{E}((\nu))$ and $\nu^{\prime} \geq \nu$

$$
\left(\partial_{J_{1}} \ldots \partial_{J_{a}} M_{v}\right)(0)=\left(\partial_{J_{1}} \ldots \partial_{J_{a}} M_{v^{\prime}}\right)(0) .
$$

Hence, from this and (5.28) it follows that for $0 \leq a \leq \nu-1, \mathbb{I}_{1}, \cdots, \mathbb{I}_{a} \in \mathbb{E}((\nu))$ and $\nu^{\prime} \geq \nu$,

$$
\left(\partial_{I_{1}} \cdots \partial_{I_{a}} \operatorname{det} M_{\nu}\right)(0)=\left(\partial_{I_{1}} \cdots \partial_{I_{a}} \operatorname{det} M_{\nu^{\prime}}\right)(0) \text {. }
$$

In view of the explicit expression of $\partial_{I_{1}} \cdots \partial_{I_{a}} \frac{1}{\operatorname{det} M_{v}}$ in terms of $\partial_{J_{1}} \cdots \partial_{J_{b}} \times$ $\operatorname{det} M_{\nu}, 1 \leq b \leq a, J_{1}, \cdots, J_{b} \in\left\{I_{1}, \cdots, I_{a}\right\}$, this implies that for $0 \leq a \leq \nu-1$, $I_{1}, \cdots, I_{a} \in \mathbb{E}((\nu))$ and $\nu^{\prime} \geq \nu$ 


$$
\left(\partial_{I_{1}} \cdots \partial_{I_{a}} \frac{1}{\operatorname{det} M_{\nu}}\right)(0)=\left(\partial_{I_{1}} \cdots \partial_{I_{a}} \frac{1}{\operatorname{det} M_{\vartheta^{\prime}}}\right)(0)
$$

We continue the discussion in the case (A). We shall present the asymptotic expansion of $\delta_{x_{0}}\left(X^{\mathrm{\varepsilon}}(1)\right)=\delta_{0}\left(X^{\varepsilon}(1)-x_{0}\right)$ in $\widetilde{D^{-\infty}}$ as $\varepsilon \downarrow 0$. Let $\nu \geq \nu_{0}$ again. Set $f_{I_{1}, \cdots, I_{a}} \in \boldsymbol{R}^{1}, I_{1}, \cdots, I_{a} \in \mathbb{E}(\infty), a \geq 1$ by

$$
f_{I_{1}, \cdots, I_{a}}:=\left(\partial_{I_{1}} \cdots \partial_{I_{a}} \frac{1}{\operatorname{det} M_{\left\|I_{1}\right\|+\cdots+\left\|I_{a}\right\|+1}}\right)(0),
$$

and define $\xi_{a} \in D^{\infty}, a \geq 0$ by

$$
\left\{\begin{array}{l}
\xi_{0}:=1, \\
\xi_{a}:=\sum_{l=1}^{a} \frac{1}{l !} \sum_{\substack{\left.I_{1}, \cdots, I_{l} \in G((\infty))\right) \\
\left\|I_{1}\right\|+\cdots+\left\|I_{l}\right\|=a}} f_{I_{1}, \cdots, I_{l}} U_{1}^{I_{1}} \cdots U_{1^{l}}^{I_{l}} \quad a \geq 1 .
\end{array}\right.
$$

Then, from (5.29) and the definition of $\xi_{a}^{((v))}$, we see that

$$
\xi_{a}^{((\nu))}=\xi_{a} \quad \text { for any } \quad 0 \leq a \leq \nu-\nu_{0} .
$$

Hence, by virtue of this and (5.24), (5.27) implies that

$$
\begin{array}{r}
\chi_{\nu}^{\varepsilon} \cdot \delta_{0}\left(F_{\nu}^{\varepsilon}(1)\right)=\varepsilon^{-N}|\operatorname{det} \beta|^{-1}\left(\Xi_{0}+\sum_{a=1}^{\nu-\nu_{0}} \varepsilon^{a} \sum_{\substack{b, c \geq 0 \\
b+c=a}} \xi_{b} \cdot \Xi_{c}\right)+O\left(\varepsilon^{\nu-\nu_{0}+1-N}\right) \\
\mathbb{D}^{-\infty} \text { as } \varepsilon \downarrow 0,
\end{array}
$$

that is, there exists an $s=s(\nu)>0$ such that for any $p>1$

$$
\begin{aligned}
& \left\|\chi_{\nu}^{\varepsilon} \cdot \delta_{0}\left(F_{\nu}^{\varepsilon}(1)\right)-\varepsilon^{-N}|\operatorname{det} \beta|^{-1}\left(\Xi_{0}+\sum_{a=1}^{\nu-\nu_{0}} \varepsilon^{a} \sum_{\substack{b, c \geq 0 \\
b+c=a}} \xi_{b} \cdot \Xi_{c}\right)\right\|_{p,-s} \\
& =O\left(\varepsilon^{\nu-\nu_{0}+1-N}\right) \quad \text { as } \quad \varepsilon \downarrow 0 .
\end{aligned}
$$

Thus, combining this and (5.12), we have that for any $p>1$

$$
\begin{aligned}
& \left\|\delta_{x_{0}}\left(X^{\varepsilon}(1)\right)-\varepsilon^{-N}|\operatorname{det} \beta|^{-1}\left(\Xi_{0}+\sum_{a=1}^{\nu-\nu_{0}} \varepsilon^{a} \sum_{\substack{b, c \geq 0 \\
b+c=a}} \xi_{b} \cdot \Xi_{c}\right)\right\|_{p_{0}-\left(s \vee_{2}([d / 2]+2)\right)} \\
& =O\left(\varepsilon^{\left.\left(l_{y}+N\right) \wedge\left(\nu-v_{0}+1\right)-N\right)}\right) \text { as } \varepsilon \downarrow 0 .
\end{aligned}
$$

Consequently, noting that by $(5.11)(\mathrm{i}),\left(l_{v}+N\right) \wedge\left(\nu-\nu_{0}+1\right)$ tends to infinity as $\nu \uparrow \infty$, we obtain the following theorem:

Theorem 5.31. Let $V_{0}, V_{1}, \cdots, V_{n} \in C_{b}^{\infty}\left(\boldsymbol{R}^{d}, \mathbb{R}^{d}\right)$ and $x_{0} \in \mathbb{R}^{d}$. Let $X^{\mathrm{q}}\left(t, x_{0}\right)$ denote the unique solution of (4.1) (for these $V_{i}, i=0,1, \cdots, n$ ) starting at $x_{0}$. Suppose that the condition (4.7) is satisfied at $x_{0}$ and that $\mu_{0}$ defined by (5.21) is 
less than or equal to 2. Then $\delta_{x_{0}}\left(X^{\varepsilon}\left(1, x_{0}\right)\right)$ has the following asymptotic expansion in $\widetilde{\mathbb{D}^{-\infty}}$ as $\varepsilon \downarrow 0$ :

and $\Psi_{a} \in \widetilde{\mathbb{D}^{-\infty}}, a \geq 0$ are given by

$$
\delta_{x_{0}}\left(X^{\varepsilon}\left(1, x_{0}\right)\right) \sim \varepsilon^{-N}|\operatorname{det} \beta|^{-1} \sum_{a=0}^{\infty} \varepsilon^{a} \Psi_{a}
$$

$$
\Psi_{0}=\Xi_{0}, \quad \Psi_{a}=\sum_{\substack{b, c \geq 0 \\ b+c=a}} \xi_{b} \circ \Xi_{c} \quad a \geq 1
$$

Here $N, \beta, \xi_{a}$ and $\Xi_{a}$ are defined by (5.14), (5.2), (5.30) and (5.23), respectively.

Second, we consider the case (B). In this case, we observe by (5.22) that

$$
\begin{aligned}
b^{\varepsilon}: & =\varepsilon^{\mu_{0}-2} a^{\varepsilon}=\varepsilon^{\mu_{0}-2} \sum_{a=1}^{\mu_{0}} \varepsilon^{2-a}\left(\delta_{a}^{\|I\| \gamma} \gamma^{I(0)}\right)_{I \in H} \\
& =\sum_{a<\mu_{0}} \varepsilon^{\mu_{0}-a}\left(\delta_{a}^{\|I\|} \gamma^{I(0)}\right)_{I \in \mathbb{H}}+\left(\delta \mu_{0} I \| \gamma^{I(0)}\right)_{I \in H} \\
& \rightarrow\left(\delta \mu_{0}^{\|I\|} \gamma^{I(0)}\right)_{I \in H}=: b \neq 0 \quad \text { as } \varepsilon \downarrow 0 .
\end{aligned}
$$

So we can take an $\varepsilon_{1}>0$ such that

$$
\left|b^{\varepsilon}\right|>\frac{1}{2}|b|>0 \quad \text { for any } \quad 0<\varepsilon \leq \varepsilon_{1} .
$$

By (5.20) and (5.17),

$$
\begin{gathered}
\chi_{\nu}^{\varepsilon} \circ \delta_{0}\left(F_{\nu}^{3}(1)\right)=\varepsilon^{-N}|\operatorname{det} \beta|^{-1} f_{\nu}\left(T_{(\nu))}^{((\varepsilon))} U_{1}^{(\nu))}\right) \delta_{-\varepsilon^{-\left(\mu_{0}-2\right)} b^{\varepsilon}}\left(\hat{A}_{(\nu))}^{\varepsilon} \hat{U}_{1}^{((\nu))}\right) \\
0<\varepsilon \leq \varepsilon_{0} .
\end{gathered}
$$

Recall that $\hat{A}_{((v))}^{\mathrm{z}} \hat{U}_{1}^{((\nu))}$ is uniformly non-degenerate. From this fact, the following is easily seen: For any $p>1$ and $m \geq 1$,

$$
\sup _{v \in \mathbb{R}^{d}} \sup _{0<\varepsilon \leq \varepsilon_{0}}\left(1+|v|^{2}\right)^{m}\left\|\delta_{v}\left(\hat{A}_{(\nu))}^{\varepsilon} \hat{U}_{1}^{(\nu))}\right)\right\|_{p_{0}-2([d / 2]+-1)}<+\infty .
$$

Hence, combining this with (5.32) and (5.33), we see that for any $p>1, m \geq 1$ and $0<\varepsilon \leq \varepsilon_{0} \wedge \varepsilon_{1}$

$$
\begin{aligned}
& \left\|\chi_{\nu}^{\varepsilon} \circ \delta_{0}\left(F_{\nu}^{\varepsilon}(1)\right)\right\|_{p,-2([d / 2]+1)} \\
& \leq \varepsilon^{-N}|\operatorname{det} \beta|^{-1} C_{p, d}\left\|f_{\nu}\left(T_{((\nu))}^{((\varepsilon))} U_{1}^{((\nu))}\right)\right\|_{2 p, 2([d / 2]+1)}
\end{aligned}
$$

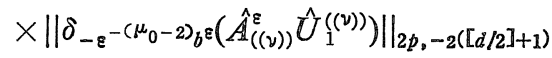

$$
\begin{aligned}
& \leq \varepsilon^{2 m\left(\mu_{0}-2\right)-N}|\operatorname{det} \beta|^{-1}\left(\frac{2}{|b|}\right)^{2 m} C_{p, d} \sup _{0<\varepsilon \leq \varepsilon_{0}}\left\|f_{\vartheta}\left(T_{((\nu))}^{((z))} U_{1}^{((\nu))}\right)\right\|_{2 p_{2}([d / 2]+1)} \\
& \times \sup _{v \in \mathbb{R}^{d}} \sup _{0<\varepsilon \varepsilon_{0}}\left(1+|v|^{2}\right)^{m}\left\|\delta_{v}\left(\hat{A}_{((v))}^{\varepsilon} \hat{U}_{1}^{((v))}\right)\right\|_{2 p_{0}-2([d / 2]+1)} .
\end{aligned}
$$

This asserts that $\left\|\chi_{\nu}^{\varepsilon} \cdot \delta_{0}\left(F_{\nu}^{\varepsilon}(1)\right)\right\|_{p,-2([d / 2]+1)}=O\left(\varepsilon^{m}\right)$ as $\varepsilon \downarrow 0$ for any $p>1$ and 
$m \geq 1$.Thus, putting this and (5.12) together, we obtain the following theorem:

Theorem 5.34. Let $V_{0}, V_{1}, \cdots, V_{n} \in C_{b}^{\infty}\left(\mathbb{R}^{d}, \mathbb{R}^{d}\right)$ and $x_{0} \in \mathbb{R}^{d}$. Let $X^{\mathrm{g}}\left(t, x_{0}\right)$ denote the unique solution of (4.1) starting at $x_{0}$. Suppose that the condition (4.7) is satisfied at $x_{0}$ and that $\mu_{0}$ is more than 2. Then it holds that for any $p>1$ and $m \geq 1$

$$
\left\|\delta_{x_{0}}\left(X^{\varepsilon}\left(1, x_{0}\right)\right)\right\|_{p,-2([d / 2]+1)}=O\left(\varepsilon^{m}\right) \quad \text { as } \varepsilon \downarrow 0
$$

\section{§6. Diagonal Short Time Asymptotics}

Suppose that $V_{0}, V_{1}, \cdots, V_{n} \in C_{b}^{\infty}\left(\mathbb{R}^{d}, \mathbb{R}^{d}\right)$ satisfy the condition (4.7) at $x_{0} \in \mathbb{R}^{d}$. Let $X^{q}\left(t, x_{0}\right)$ be the unique solution of (4.1) for $V_{i}, i=0,1, \cdots, n$ starting at $x_{0} \in \mathbb{R}^{d}$. By (4.8),

$$
p\left(\varepsilon^{2}, x_{0}, x_{0}\right)=E\left[\delta_{x_{0}}\left(X^{\varepsilon}\left(1, x_{0}\right)\right),\right.
$$

where $p(t, x, y)$ is the fundamental solution of $\frac{\partial}{\partial t}=\frac{1}{2} \sum_{i=1}^{n} \hat{V}_{i}^{2}+\hat{V}_{0}$. In this section, we study the short time asymptotic of $p\left(t, x_{0}, x_{0}\right)$. Let $\nu_{0}$ be the smallest integer $\nu$ satisfying (4.7) at $x_{0}$ and let $\mu_{0}$ be a nonnegative integer defined by (5.21).

First, in the case (B), i.e., the case where $\mu_{0} \geq 3$, we easily see from (6.1) and Theorem (5.34) that

$$
p\left(t, x_{0}, x_{0}\right)=O\left(t^{m}\right) \quad \text { as } t \downarrow 0 \quad \text { for any } m \geq 1 \text {. }
$$

Next, as to the case (A), i.e., the case where $\mu_{0} \leq 2$. By (6.1) and Theorem (5.31), we have obviously

$$
p\left(\varepsilon^{2}, x_{0}, x_{0}\right) \sim \varepsilon^{-N}|\operatorname{det} \beta|^{-1} \sum_{a=0}^{\infty} \varepsilon^{a} E\left[\Psi_{a}\right] \quad \text { as } \varepsilon \downarrow 0 .
$$

Recall (3.12) (ii). From this fact, we easily see that

$$
\left\{\begin{array}{l}
\text { (i) } \xi_{a}(-w)=(-1)^{a} \xi_{a}(w) \quad a \geq 0, \\
\text { (ii) } v_{a}(-w)=(-1)^{a} T_{H}^{((-1))} v_{a}(w) \quad a \geq 0,
\end{array}\right.
$$

where $T_{\boldsymbol{H}}^{(\lambda))} \in \operatorname{Hom}\left(\mathbb{R}^{d}, \mathbb{R}^{d}\right)$ is defined by $T_{H}^{((\lambda))}\left(\left(v^{I}\right)_{I \in \boldsymbol{H}}\right):=\left(\lambda\|I\|_{\boldsymbol{v}^{I}}\right)_{I \in \boldsymbol{H}^{*}}$. Here, note that in general, it holds that for $l \geq 0, I_{1}, \cdots, I_{l} \in \mathbb{H}$ and $v \in \mathbb{R}^{d}$

$$
\left(\partial_{I_{1}} \cdots \partial_{I_{l}} \delta_{v}\right)\left(T_{H}^{(\lambda))} v_{0}\right)=|\lambda|^{-N} \lambda^{-\left(|| I_{1}||+\cdots+|| I_{l} \|\right)}\left(\partial_{I_{1}} \cdots \partial_{I_{l}} \delta_{T_{H Z}^{(\lambda-1)}}^{\left.(\lambda)_{v}\right)}\left(v_{0}\right) \quad \lambda \neq 0 .\right.
$$

Hence, combining this with (6.3) (ii), we also see that 


$$
\Xi_{a}(-w)=(-1)^{a} \Xi_{a}(w) \quad a \geq 0 .
$$

Thus, from this and (6.3) (i), it follows that $\Psi_{a}(-w)=(-1)^{a} \Psi_{a}(w)$ for $a \geq 0$, and as its consequence, we have that

$$
E\left[\Psi_{a}\right]=0 \quad \text { if } a \text { is odd },
$$

because the mapping $w \mapsto-w$ preserves the measure $\mathbb{P}$. Therefore, by (6.2) and (6.4), we obtain that

$$
p\left(t, x_{0}, x_{0}\right) \sim t^{-N / 2} \sum_{b=0}^{\infty}|\operatorname{det} \beta|^{-1} E\left[\Psi_{2 b}\right] t^{b} \quad \text { as } t \downarrow 0 .
$$

It remains to study the positivity of the first constant $c_{0}$ appearing in this asymptotic expansion, that is, the positivity of $E\left[\delta_{0}\left(v_{0}\right)\right]$. But, under the case where $\mu_{0} \leq 2$ only, we are not able to show that. For this, we consider the following strong condition $(\mathrm{A})^{\prime}$ or $(\mathrm{A})^{\prime \prime}$ :

(A) $\left\{\begin{array}{l}\text { On some neighborhood } W \text { of } x_{0}, \hat{V}_{0} \text { is represented as } \hat{V}_{0}=\sum_{i=1}^{n} g^{i} \hat{V}_{i} \\ \text { where } g^{i} \in C^{\infty}(W), \quad 1 \leq i \leq n .\end{array}\right.$ $(\mathrm{A})^{\prime \prime} \quad \nu_{0} \leq 3$ and $\mu_{0} \leq 2$.

In what follows, under the condition $(\mathbb{A})^{\prime}$ or $(\mathbb{A})^{\prime \prime}$, we shall show the positivity of $E\left[\delta_{0}\left(v_{0}\right)\right]:$ First of all, recall $v_{0} \in \mathbb{D}^{\infty}\left(\mathbb{R}^{*} \mathbb{H}\right)$ given in (5.23):

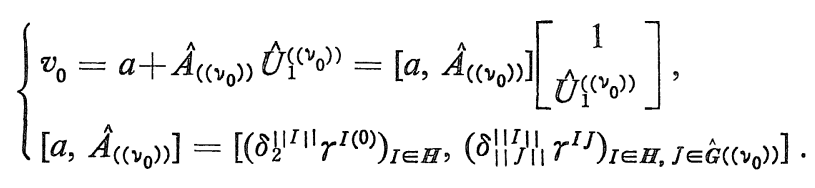

First we consider the case $(\mathrm{A})^{\prime}$. Set $\mathbb{E}^{0}(\nu):=\{I \in \mathbb{E}(\nu) ; \alpha(I)=0\}$ and $\mathbb{G}^{0}(\nu):=\mathbb{E}^{0}(\nu) \cap \mathbb{G}(\nu)$. In this case, it is easy to see that for each $I \in \mathbb{E}(\infty)$, there exist $g^{I J} \in C^{\infty}(W), J \in \mathbb{E}^{0}(|I|)$ such that

$$
\hat{V}_{[I]}=\sum_{J \in \mathbb{E}^{0}(|I|)} g^{I J} \hat{V}_{[J]} \quad \text { on } W .
$$

And, we can see from (6.6) that for each $\nu \geq 1$

$$
\text { l.1. }\left\{\hat{V}_{[I]}\left(x_{0}\right) ; \mathbb{I} \in \hat{G}((\nu))\right\}=\text { l.1. }\left\{\hat{V}_{[I]}\left(x_{0}\right) ; \mathbb{I} \in \mathbb{G}^{0}(\nu)\right\} \text {. }
$$

Since, in particular, it follows from this that

$$
T_{x_{0}}\left(\mathbb{R}^{d}\right)=\text { l.1. }\left\{\hat{V}_{[I]}\left(x_{0}\right) ; \mathbb{I} \in \mathbb{G}^{0}\left(\nu_{0}\right)\right\},
$$

an $\mathbb{H}$ appearing in (5.1) can be chosen in $\mathbb{G}^{0}\left(\nu_{0}\right): \mathbb{H} \subset \mathbb{G}^{0}\left(\nu_{0}\right)$. From (6.6) and this choice of $\mathbb{H},(5.3)$ is rewritten as follows: 


$$
\left\{\begin{aligned}
\text { (i) } \quad r^{I J}=\delta_{F}^{I} & \text { if } J \in \mathbb{H}, \\
\text { (ii) } \quad r^{I J}=0 & \text { if }|I| \geq|J|+1 .
\end{aligned}\right.
$$

Hence, combining (6.7) (ii) with (6.5), we have that

$$
v_{0}=\left(\delta_{|J|}^{I} \mid \gamma^{I J}\right)_{I \in H, J \in G^{0}\left(v_{0}\right)}\left(U_{1}^{J}\right)_{J \in G^{0}\left(v_{0}\right)} .
$$

Note that rank $\left[\left(\delta_{|J|}^{I} \mid \gamma^{I J}\right)_{I \in H, J \in G^{0}\left(v_{0}\right)}\right]=H H=d$ by (6.7) (i). Thus, from this fact and Proposition (A.13), the positivity of $E\left[\delta_{0}\left(v_{0}\right)\right]$ follows immediately.

The positivity of $E\left[\delta_{0}\left(v_{0}\right)\right]$ in the case $(\mathrm{A})^{\prime \prime}$ can be shown in the same way as above by noting that $\operatorname{rank} \hat{A}_{\left(\left(v_{0}\right)\right)}=\mathbb{H} H=d$.

Consequently, summarizing all the above, we have the following theorem:

Theorem 6.8. The short time asymptotic of $p(t, x, y)$ at the diagonal $\left(x_{0}, x_{0}\right)$ is as follows:

(i) If $\mu_{0} \leq 2$, then

$$
p\left(t, x_{0}, x_{0}\right) \sim t^{-N / 2} \sum_{b=0}^{\infty} c_{b} t^{b} \quad \text { as } t \downarrow 0
$$

with $c_{b}=|\operatorname{det} \beta|^{-1} E\left[\Psi_{2 b}\right], b \geq 0$. Here $N$ and $\beta$ are defined by (5.14) and (5.2), respectively, and $\Psi_{a}$ is given in Theorem (5.31). Further, if either the condition (A)' or $(\mathrm{A})^{\prime \prime}$ holds, then $c_{0}$ is positive.

(ii) If $\mu_{0} \geq 3$, then $p\left(t, x_{0}, x_{0}\right)=O\left(t^{m}\right)$ as $t \downarrow 0$ for any $m \geq 1$.

The condition $(\mathrm{A})^{\prime}$ on $\hat{V}_{0}$ is just one in Kusuoka-Stroock [10], and it clearly contains the case where $\hat{V}_{0} \equiv 0$, which was treated by Léandre [11] and Ben Arous [1]. Also, in the elliptic case, i.e., the case where $\hat{V}_{1}\left(x_{0}\right), \cdots, \hat{V}_{n}\left(x_{0}\right)$ span the tangent space at $x_{0}$, this condition is automatically satisfied for any $\hat{V}_{0}$. The following is a simple example satisfying the condition $(\mathrm{A})^{\prime \prime}$ but not the condition $(\mathrm{A})^{\prime}$ :

Example. (Kolmogorov [6]). Let $d=2, n=1$ and $V_{1}=\left[\begin{array}{l}1 \\ 0\end{array}\right], V_{0}=\left[\begin{array}{l}0 \\ x^{1}\end{array}\right]$. Then it is easily checked that for every $x=\left[\begin{array}{l}x^{1} \\ x^{2}\end{array}\right] \in \mathbb{R}^{2}$, (i) the condition $(\mathrm{A})^{\prime}$ is not satisfied, (ii) $N(x)=4, \nu_{0}(x)=3$ and

$$
\mu_{0}(x)= \begin{cases}0 & \text { if } x^{1}=0 \\ 3 & \text { if } x^{1} \neq 0\end{cases}
$$

Hence, by applying Theorem (6.8), we can obtain the diagonal short time asymptotic of the heat kernel $p(t, x, y)$ of $\frac{\partial}{\partial t}=\frac{1}{2} \frac{\partial^{2}}{\partial\left(x^{1}\right)^{2}}+x^{1} \frac{\partial}{\partial x^{2}}$. But, in this case, 
$p(t, x, x)$ is concretely evaluated:

$$
p(t, x, x)=\frac{\sqrt{3}}{\pi} \frac{1}{t^{2}} \exp \left(-\frac{6\left(x^{1}\right)^{2}}{t}\right) \quad t>0, x=\left[\begin{array}{c}
x^{1} \\
x^{2}
\end{array}\right] .
$$

So, when $x^{1} \neq 0$, Theorem (6.8) (ii) is, for example, restated as follows:

$$
\lim _{t: 0}-t \log p(t, x, x)=6\left(x^{1}\right)^{2}>0 .
$$

This example suggests to us that Theorem (6.8) (ii) has room for improvement.

\section{Appendix}

First of all, following Kusuoka-Stroock [9], we introduce the following notations for $I=\left(i_{1}, \cdots, i_{a}\right) \in \mathbb{E}(\infty)$ :

$$
I_{*}:=i_{a}, \quad I^{\prime}:=\left\{\begin{array}{l}
\phi \quad \text { if } a=1 \\
\left(i_{1}, \cdots, i_{a-1}\right) \quad \text { if } a \geq 2 .
\end{array}\right.
$$

Let us fix $\nu \geq 1$ and let $\mathbb{Z} \subset \mathbb{E}(\nu)$ be a non-empty set satisfying the following:

$$
\left\{\begin{array}{l}
\text { If }\left(i_{1}, \cdots, i_{a}\right) \in \mathbb{Z}, \text { then }\left(i_{1}, \cdots, i_{b}\right),\left(i_{b+1}, \cdots, i_{a}\right) \in \mathbb{I} \text { for any } \\
1 \leq b \leq a-1, \text { and }\left(i_{\sigma(1)}, \cdots, i_{\sigma(a)}\right) \in \mathbb{I} \text { for any permutation } \sigma \in \mathbb{S}_{a} .
\end{array}\right.
$$

Putting $\mathbb{Z}$ in place of $\mathbb{E}(\nu)$, we trace our discussions in $\S 1, \S 2$ and $\S 3$ : Set

$$
\mathbb{G}^{I}(\nu):=\mathbb{I} \cap \mathbb{G}(\nu), \quad \hat{G}^{I}(\nu):=\mathbb{G} I(\nu) \backslash\{(0)\} .
$$

Note that

$$
\mathbb{G}^{I}(\nu)=\hat{G}^{I}(\nu) \quad \text { if }(0) \notin \mathbb{I} .
$$

As before, we identify

$$
\left\{\left(y^{I}\right)_{I \in I} ; y^{I} \in \mathbb{R}^{1}, I \in \mathbb{I}\right\} \simeq \mathbb{R}^{\mathbb{R}},
$$

and the coordinate system on $\mathbb{R}^{\sharp I}$ is also denoted by $y^{I}, I \in \mathbb{I}$. We understand $\mathbb{R}^{\sharp G^{I}(\nu)}$ and $\mathbb{R}^{\sharp G^{I}(v)}$ similarly to $\mathbb{R}^{\sharp I}$. Define $Q_{i}^{I} \in \mathfrak{X}\left(\mathbb{R}^{\# I}\right), i \in \mathbb{E}$ by

$$
Q_{i}^{I}:=\left\{\begin{array}{cl}
\sum_{I \in \mathbb{I} ; I_{*}=i} y^{I^{\prime}} \frac{\partial}{\partial y^{I}} & \text { if }(i) \in \mathbb{Z} \\
0 & \text { if }(i) \notin \mathbb{I}
\end{array}\right.
$$

where $y^{\phi}:=1$ for convenience, and denote by $g_{I}$ the Lie subalgebra of $\mathfrak{X}\left(\mathbb{R}^{\mathbb{R}}\right)$ generated by $Q_{i}^{I}, i \in \mathbb{E}$. Then it holds that 


$$
\left\{\begin{array}{l}
\mathbb{R}^{\sharp G^{I}(\nu)} \text { can be regarded as a Lie group with a multiplication } \times \\
\text { defined by means of the Campbell-Hausdorff formula, and if } \mathfrak{h}_{I} \\
\text { denotes the right invariant Lie algebra of } \mathbb{R}^{\sharp} G^{I(\nu)}, \text { then } \mathfrak{g}_{I} \text { is } \\
\text { isomorphic to } \mathfrak{h}_{I} \text { under the correspondence } Q_{i}^{I} \leftrightarrow R_{i}^{I} .
\end{array}\right.
$$

Here

$$
R_{i}^{I}:= \begin{cases}\text { an element of } \mathfrak{h}_{I} \text { such as } R_{i}^{I}(0)=\left(\frac{\partial}{\partial u^{i}}\right)_{0} & \text { if }(i) \in \mathbb{I}, \\ 0 & \text { if }(i) \notin \mathbb{I} .\end{cases}
$$

Since $Q_{[I]}^{I}=0$ for $I \in \mathbb{E}(\infty) \backslash \mathbb{I}$ and $\left(\Pi_{I}^{\nu}\right) * Q_{i}^{(\nu)}=Q_{i}^{I}$, where $\Pi_{I}^{\nu} \in \operatorname{Hom}\left(\mathbb{R}^{\sharp E(\nu)}, \mathbb{R}^{\sharp I}\right)$ is a projection to $\mathbb{R}^{\sharp I}$, the following follows from (A.2):

$$
\begin{aligned}
& R_{[I]}^{I}=0 \quad \text { for } I \in \mathbb{E}(\infty) \backslash \mathbb{I} . \\
& \left(P_{I}^{\nu}\right)_{*} R_{[I]}^{(\nu)}=R_{[I]}^{I} \quad \text { for any } I \in \mathbb{E}(\infty),
\end{aligned}
$$

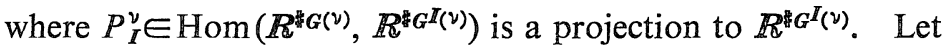

$$
\begin{aligned}
& \mathfrak{b}_{I}:=\text { the Lie subalgebra generated by } R_{i}^{I}, i \in\{1, \cdots, n\}, \\
& \mathfrak{l}_{I}:=\text { the Lie subalgebra generated by } R_{i}^{I}, i \in\{0,1, \cdots, n\}, \\
& \mathfrak{\mathfrak { I }}_{I}:=\text { the ideal in } \mathfrak{I}_{I} \text { generated by } R_{i}^{I}, i \in\{1, \cdots, n\} .
\end{aligned}
$$

Then it is easy to see that

$$
\left\{\begin{aligned}
& \text { (i) } \mathfrak{l}_{I}=\mathfrak{b}_{I}, \\
& \text { (ii) } \mathfrak{b}_{I}=\mathfrak{l}_{I}=\mathfrak{\mathfrak { t }}_{I} \quad \text { if }(0) \notin \mathbb{I}, \\
& \text { (iii) } \operatorname{dim} \mathfrak{\mathfrak { t }}_{I}=\sharp \hat{G}^{I}(\nu)=\$ \mathbb{G}^{I}(\nu)-1 \quad \text { if }(0) \in \mathbb{I} .
\end{aligned}\right.
$$

Next, recalling $U_{t}^{(\infty)}=\left(U_{t}^{I}\right)_{I \in G(\infty)}$ introduced in $\S 3$, we define

$$
U_{t}^{I}:=\left(U_{t}^{I}\right)_{I \in G^{I}(v)}, \quad \hat{U}_{t}^{I}:=\left(U_{t}^{I}\right)_{I \in \hat{G}^{I}(v)} .
$$

By (A.1) and (3.6), it is easy to see that

$$
U_{t}^{I}= \begin{cases}\hat{U}_{t}^{I} & \text { if }(0) \notin \mathbb{I} \\
{\left[\begin{array}{c}
t \\
U_{t}^{I}
\end{array}\right]} & \text { if }(0) \in \mathbb{I} .\end{cases}
$$

Since $U_{t}^{I}=P_{I}^{\nu} U_{t}^{(v)}$, by combining (A.4) and (3.2), $U_{t}^{I}$ is the unique solution of

$$
\left\{\begin{array}{l}
d U_{t}=\sum_{i \in E} R_{i}^{I}\left(U_{t}\right) \circ d w_{t}^{i} \\
U_{0}=0 .
\end{array}\right.
$$

Generally, for each $u \in \mathbb{R}^{z} G I(v), U_{t}^{I} \times u$ is the unique solution of the above SDE 
starting at $u$. So, from this fact, we see that for any $t, s \geq 0$

$$
\text { (1.A) } P\left(U_{t+s}^{I} \in d u\right)=\int_{\mathbb{R}^{i} G^{I}(v)} P\left(U_{t}^{T} \in d u \times(-v)\right) P\left(U_{s}^{F} \in d v\right) \quad u \in \mathbb{R}^{\mathbb{R} G^{I}(\nu)}
$$

On the other hand, by Proposition (3.8), $\hat{U}_{1}^{I}\left(\in \mathbb{Z}^{\infty}\left(\mathbb{R}^{\left.\left.\frac{f \hat{G}^{K}(\nu)}{}\right)\right)}\right.\right.$ is non-degenerate in the Malliavin sense, and generally so is $\hat{U}_{t}^{I}$ for $t>0$. Hence, for each $t>0$, $\widehat{U} \frac{I}{t}$ has a smooth density $p_{t}$ with respect to the Lebesgue measure on $\mathbb{R}^{\# \hat{G}^{I}(\nu)}$ :

$$
P\left(\hat{U}_{t}^{I} \in d \hat{u}\right)=p_{\hat{t}}(\hat{u}) d \hat{u}, \quad \hat{u} \in \mathbb{R}^{m} \hat{G}^{\bar{I}}(\nu) .
$$

Thus, combining this with (A.6), we have

$$
P\left(U_{t}^{I} \in d u\right)= \begin{cases}p_{i}(u) d u & \text { if }(0) \notin \mathbb{I}, \\ \delta_{\hat{\imath}}\left(d u^{0}\right) p_{t}(\hat{u}) d \hat{\imath} & \text { if }(0) \in \mathbb{I},\end{cases}
$$

where $u=\left[\begin{array}{l}u^{0} \\ \hat{u}\end{array}\right], u^{0} \in \mathbb{R}^{1}, \hat{u} \in \mathbb{R}^{*} \hat{G}^{I}(v)$, for $u \in \mathbb{R}^{*} G^{I}(\nu)$.

We further view the case when $(0) \notin \mathbb{I}$. In this case, from (A.7) and (A.8), we easily see that for any $i, s>0$ and $u \in \mathbb{R}^{: G^{I}(\nu)}$,

$$
p_{\imath+s}(u)=\int_{\mathbb{R}^{\sharp G} I^{I}(\nu)} p_{t}(u \times(-v)) p_{s}(v)\left|\operatorname{det} \partial_{u}(u \ltimes(-v))\right| d v .
$$

On the other hand, from (A.5) (i), (ii), the support of $P\left(U_{i}^{I} \in 0\right)$ coincides with $\mathbb{R}^{\sharp G^{I}(\nu)}$ for $t>0$ (cf. [7], [14]). Hence, from this and (A.9), it follows immediately that $p_{t}(u)>0$ for any $t>0$ and $u \in \mathbb{R}^{3} G^{\mathbb{I}}(\nu)$.

Next we view the case when $(0) \in \mathbb{H}$. To this end, define a smooth function $f: \mathbb{R}^{1} \times \mathbb{R}^{1} \times \mathbb{R}^{\hat{G} \hat{G}^{I}(\nu)} \times \mathbb{R}^{\sharp ! \hat{G}^{I}(\nu)} \rightarrow \mathbb{R}^{\mathbb{R}^{\hat{G}} \hat{G}^{I}(\nu)}$ such that the following holds: For $u^{0}, v^{0} \in \mathbb{R}^{1}$ and $\hat{u}, \hat{v} \in \mathbb{R}^{*} \hat{G}^{I}(\nu)$

$$
\left[\begin{array}{l}
u^{0} \\
\hat{u}
\end{array}\right] \times\left[\begin{array}{l}
v^{0} \\
\hat{v}
\end{array}\right]=\left[\begin{array}{c}
u^{0}+v^{0} \\
f\left(u^{0}, v^{0}, \hat{u}, \hat{v}\right)
\end{array}\right]
$$

Then it is easily seen that

(i) for fixed $u^{0}, v^{0} \in \mathbb{R}^{1}$ and $\hat{u} \in \mathbb{R}^{\sharp \hat{G}^{I}(\nu)}, \int\left(u^{0}, v^{0}, \hat{u}, \circ\right)$ is diffeomorphic,

(ii) for fixed $u^{0}, v^{0} \in \mathbb{R}^{1}$ and $\hat{v} \in \mathbb{R}^{\sharp \hat{G}^{I}(\nu)}, f\left(u^{0}, v^{0}, \cdot, \hat{v}\right)$ is also diffeomorphic.

Further, putting (A.7), (A.8) and (A.10) together, we see that for any $i, s>0$ and $\hat{u} \in \mathbb{R}^{2} \hat{G}^{R}(v)$

(A.11) $p_{t+s}(\hat{u})=\int_{\mathbb{R}^{\sharp} \hat{G}^{I}(v)} p_{t}(f(t+s,-s, \hat{u},-\hat{v})) p_{s}(\hat{v})\left|\operatorname{det} \partial_{\hat{\imath} \hat{\jmath}}(t+s,-s, \hat{u},-\hat{v})\right| d \hat{v}$.

On the other hand, if we assume that 


$$
\left[\mathfrak{b}_{I}, \mathfrak{i}_{I}\right](u) \subset \mathfrak{b}_{I}(u) \quad \text { for every } u \in \mathbb{R}^{\sharp} G^{I(v)},
$$

then, by applying Theorem 6.1 in Kunita [7], this together with (A.5) (i), (iii) implies that the support of $P\left(\hat{U}_{t}^{I} \in \bullet\right)$ coincides with $\mathbb{R}^{\sharp \hat{G}^{I}(v)}$. Hence, under the assumption (A.12), it follows from this and (A.11) that $p_{t}(\hat{u})>0$ for any $t>0$ and $\hat{u} \in \mathbb{R}^{\hat{k} \hat{G} T^{I}(\nu)}$.

$\mathbb{E}^{0}(\nu):=\{I \in \mathbb{E}(\nu) ; \alpha(I)=0\}$ is in the case when $(0) \notin \mathbb{Z} ; \mathbb{E}((2))$ and $\mathbb{E}((3))$ are in the case when $(0) \in \mathbb{H}$, and by virtue of (A.3), in these cases, the assumption (A.12) is satisfied. Therefore, from the above, we can state the following:

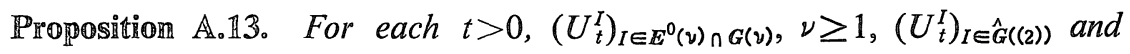
$\left(U_{t}^{I}\right)_{I \in \hat{G}((3))}$ have positive smooth densities.

\section{Acknowledgment}

The author wishes to express sincere gratitude to Professor S. Watanabe and Professor S. Kotani for their encouragement.

\section{References}

[1] Ben Arous, G., Développement asymptotique du noyau de la chaleur hypoelliptique sur la diagonale, preprint.

[2] Bismut, J.M., Large deviations and the Malliavin calculus, Progress in Math., 45, Birkhauser, 1984.

[3] Gaveau, B., Principe de moindre action, propagation de la chaleur et estimées souselliptiques sur certains groupes nilpotents, Acta Math., 139 (1977), 95-153.

[4] Ikeda, N. and Watanabe, S., An introduction to Malliavin's calculus, Stochastic Analysis, Proc. Taniguchi Symp., Katata 1982, 1-52, Kinokuniya, 1984.

[5] Jerrison, D.S. and Sánchez-Calle, A., Estimates for the heat kernel for a sum of squares of vector fields, preprint.

[6] Kolmogorov, A.N., Zufallige Bewegungen, Ann. Math. II, 35 (1934), 116-117.

[7] Kunita, H., Supports of diffusion processes and controllability problems, Proc. Intern. Symp. SDE Kyoto 1976 (ed. by K. Itô), 163-185, Kinokuniya, Tokyo, 1978.

[8] Kusuoka, S., The generalized Malliavin calculus based on Brownian sheet and Bismut's expansion for large deviation, Stochastic Processes-Mathematics and Physics, Proc. BiBoS Symp., Bielefeld 1984, 141-157, Lect. Notes in Math., 1158, Springer, 1986.

[9] Kusuoka, S. and Siroock, D.W., Applications of the Malliavin calculus, Part II, $J$. Fac. Sci. Univ. Tokyo, 32 (1985), 1-76.

[10] —, Applications of the Malliavin calculus, Part III, ibid. 34 (1987), 391-442.

[11] Léandre, R., Développement asymptotique de la dénsite d'une diffusion dégénérée, preprint.

[12] Rothschild, L. and Stein, E.M., Hypoelliptic differential operators and nilpotent Lie groups, Acta Math., 137 (1977), 247-320.

[13] Sánchez-Calle, A., Fundamental solutions and geometry of the sum of squares of vector fields, Invent. Math., 78 (1984), 143-160.

[14] Stroock, D.W. and Varadhan, S.R.S., On the support of diffusion processes with 
applications to the strong maximum principle, Proc. Sixth Berkeley Symp. Math. Statist. Prob. III, 333-359, Univ. California Press, Berkeley, 1972.

[15] Stroock, D.W. and Varadhan, S.R.S., Multidimensional diffusion processes, Springer, Berlin, 1979.

[16] Watanabe, S., Lectures on stochastic differential equations and Malliavin calculus, Tata Institute of Fundamental Research, 1984.

[17] - Analysis of Wiener functionals (Malliavin calculus) and its applications to heat kernels, Ann. Prob., 15 (1987), 1-39.

[18] Yamato, Y., Stochastic differential equations and nilpotent Lie algebras, Z. Wahr. verw. Geb., 47 (1979), 213-229. 
